\title{
Title: Enhanced photovoltage for inverted planar heterojunction perovskite solar cells
}

Authors: Deying Luo, ${ }^{1,2^{*}}$ Wenqiang Yang, ${ }^{1 *}$ Zhiping Wang, ${ }^{3 *}$ Aditya Sadhanala, ${ }^{4}$ Qin Hu, ${ }^{1}$ Rui

Su, ${ }^{1}$ Ravichandran Shivanna, ${ }^{4}$ Gustavo F. Trindade, ${ }^{5}$ John F. Watts, ${ }^{5}$ Zhaojian Xu, ${ }^{1}$ Tanghao

Liu, ${ }^{1}$ Ke Chen, ${ }^{1}$ Fengjun Ye,${ }^{1}$ Pan Wu, ${ }^{1}$ Lichen Zhao, ${ }^{1}$ Jiang Wu, ${ }^{1}$ Yongguang Tu, ${ }^{1}$ Yifei Zhang, ${ }_{1}$

Xiaoyu Yang, ${ }^{1}$ Wei Zhang, ${ }^{6 \dagger}$ Richard H. Friend ${ }_{2,7 \dagger}^{4}$ Qihuang Gong, ${ }^{1,2,7}$ Henry J. Snaith, ${ }^{3 \dagger}$ Rui Zhu ${ }^{1,}$

\section{Affiliations:}

${ }^{1}$ State Key Laboratory for Artificial Microstructure and Mesoscopic Physics, Department of Physics, Peking University, Beijing, 100871, China

${ }^{2}$ Collaborative Innovation Center of Quantum Matter, Beijing, 100871, China

${ }^{3}$ Clarendon Laboratory, Department of Physics, University of Oxford, Parks Road, Oxford, OX1 3PU, United Kingdom

${ }^{4}$ Cavendish Laboratory, JJ Thomson Avenue, Cambridge CB3 OHE, United Kingdom

$15{ }^{5}$ The Surface Analysis Laboratory, Faculty of Engineering and Physical Sciences, University of Surrey, Guildford GU2 7XH, United Kingdom

${ }^{6}$ Advanced Technology Institute, University of Surrey, Guildford GU2 7XH, United Kingdom

${ }^{7}$ Collaborative Innovation Center of Extreme Optics, Shanxi University, Taiyuan, Shanxi, 030006, China

*These authors contributed equally to this work

†Corresponding author: E-mail: wz0003@surrey.ac.uk (W.Z.); henry.snaith@physics.ox.ac.uk (H.J.S); iamzhurui@pku.edu.cn (R.Z.).

Abstract: The highest power conversion efficiencies (PCEs) reported for perovskite solar cells with inverted planar structures are still inferior to those of PSCs with regular structures, mainly because of lower open-circuit voltages $\left(V_{\mathrm{oc}}\right)$. Herein, we report a strategy to reduce nonradiative recombination for the inverted devices, based on a simple solution-processed secondary growth technique. This approach produces a wider bandgap top layer and a more n-type perovskite film, which mitigates nonradiative recombination, leading to an increase in $V_{\mathrm{oc}}$ by up to 100 millivolts. We achieved a high $V_{\text {oc }}$ of 1.21 volts without sacrificing photocurrent, corresponding to a voltage-deficit of 0.41 volts at a bandgap of 1.62 electron volts. This improvement leads to a stabilized power output approaching $21 \%$ at the maximum power point.

One Sentence Summary: We report a $V_{\text {oc }}$ of 1.21 volts in inverted planar heterojunction perovskite solar cells based on a solution-processed secondary growth technique. 


\section{Main Text:}

Recently, perovskite solar cells (PSCs) with inverted planar heterojunction structures, wherein a polycrystalline perovskite film is sandwiched between a hole- and an electronextraction layer, have gained attention as they offer the promise of easy fabrication, compatibility with flexible substrates, versatility of energy-band engineering, and the possibility of fabricating multijunction cells (1-4); moreover, they have achieved PCEs exceeding 20\%. Further enhancement of their PCEs is now mainly hampered by the relatively low $V_{\text {oc }}$, typically $<1.10 \mathrm{~V}$ in comparison with $>1.20 \mathrm{~V}$ reported for the regular PSCs using similar bandgap perovskites $(2,5)$. The less-than-ideal $V_{\text {oc }}$ of inverted PSCs is attributed to the nonradiative recombination losses both inside the perovskite bulk material and at the interfacial contacts (6-8), owing to the presence of a considerable density of defects or recombination centers (9-15). Critically, although "isolated" perovskite films can exhibit very high radiative efficiencies, the radiative efficiency drops considerably when perovskite films contact the charge-extraction layers $(16,17)$. Several approaches to reducing the nonradiative recombination-including increasing the grain size $(18,19)$, surface passivation (3), ion compensation (11) and heterojunction engineering $(20,21)$-have been proposed, pushing the $V_{\mathrm{oc}}$ up to $1.15 \mathrm{~V}$ in inverted PSCs. In addition, the precise origin of the higher $V_{\mathrm{oc}}$ for regular PSCs, which often include a thin mesoporous scaffold layer, is not clear. Evidence suggests that the perovskite film crystallized within this "scaffold region" may be more n-type in nature, leading to a favorable ntype contact at the perovskite n-type charge-extraction region (22-24). However, the perovskite film crystallized on a p-type substrate has been shown to be more p-type (or less n-type) in nature at this contact interface (25). These results suggest that the nature of the perovskite film, and specifically its doping state (n, p, or intrinsic) near the charge-extraction layers, are strongly influenced by the polarity of the substrates on which the films are crystallized. Consequently, controlling the nature of the perovskite film at the contact interface may be an effective way to achieve high $V_{\mathrm{oc}}$ in inverted PSCs. Here, we introduce a means to deliver a wider bandgap region near the top surface of the film and a more n-type perovskite film by using solution-processed secondary growth (SSG) technique, leading to a substantial increase in $V_{\text {oc }}$.

The SSG process includes two steps: (i) the preparation of perovskite films by solution processing and (ii) the secondary growth with the assistance of guanidinium bromide (which we abbreviate to SSG-G). We started with the preparation of a mixed-cation lead mixed-halide perovskite layer using a non-stoichiometric recipe of $\left(\mathrm{FA}_{0.95} \mathrm{PbI}_{2.95}\right)_{0.85}\left(\mathrm{MAPbBr}_{3}\right)_{0.15}(14,26)$, where MA and FA denote methylammonium and formamidinium, respectively. In Fig. 1A, we show the scanning electron microscopy (SEM) image of the control perovskite film. The bright crystals on the surface indicate regions of higher electron density, or regions, that accumulated charges during the measurement. From energy dispersive X-ray (EDX) analysis (fig. S1), we determined that the lead halide complex mainly dominated the composition of the bright crystals in the control sample, absent of carbon, so we assigned it as $\mathrm{PbI}_{1.50} \mathrm{Br}_{0.50}$. We further confirmed this via X-ray diffraction (XRD) analysis of the control sample (Fig. 1B), where we assigned the diffraction peak $\left(2 \theta=13.1^{\circ}\right)$ to $\mathrm{PbX}_{2}(\mathrm{X}$ is a mixture of $\mathrm{I}$ and $\mathrm{Br}$ at a $\sim 1.5: 0.5$ ratio), using the standard diffraction peaks of $\mathrm{PbI}_{2}$ at $2 \theta=12.67^{\circ}$ and $\mathrm{PbBr}_{2}$ at $2 \theta=14.37^{\circ}$ as the references. Previously, the presence of the $\mathrm{PbX}_{2}$ diffraction peak has been interpreted as indicating the presence of a $\mathrm{PbX}_{2}$ "shell" surrounding the perovskite grains $(26,27)$. In contrast, our XRD analysis shows that the $\mathrm{PbX}_{2}$ constitutes entirely separate grains. From the Sheerer broadening of the $\mathrm{PbX}_{2}$ peak, we estimated an average crystal grain size of $42 \mathrm{~nm}$, which was consistent with the size of the bright grains observed in the SEM images. Through SSG-G, the excess $\mathrm{PbX}_{2}$ 
crystals were "digested", and the perovskite film displayed distinct surface morphologies (Fig. $1 \mathrm{~A}$ and fig. S2). From the XRD patterns (Fig. 1B), we observed considerably reduced $\mathrm{PbX}_{2}$ diffraction peak intensity.

We evaluated the photovoltaic performances of the perovskite films with and without the SSG-G process by fabricating inverted planar heterojunction PSCs \{device structure: indium tin oxide (ITO)/poly[bis(4-phenyl)(2,4,6-trimethylphenyl)amine] (PTAA)/perovskite/[6,6]-phenyl$\mathrm{C}_{61}$-butyric acid methyl ester $\left(\mathrm{PC}_{61} \mathrm{BM}\right)$ /buckminsterfullerene $\left(\mathrm{C}_{60}\right)$ /bathocuproine $(\mathrm{BCP}) /$ copper $(\mathrm{Cu})\}$ and measuring the current density-voltage $(J-V)$ curves under simulated AM (air mass) $1.5 \mathrm{G}$ (global) illumination at $100 \mathrm{~mW} \mathrm{~cm} \mathrm{~cm}^{-2}$. We systematically optimized the processing parameters for the SSG process, variations of which we show in table S1-S5. All the devices exhibited negligible hysteresis (table S6). In Fig. 2A, we present $J$ - $V$ curves of the PSCs obtained from reverse scans. We found that the SSG-G devices delivered increased $V_{\text {oc }}$ by up to 100 millivolts $(\mathrm{mV})$ compared with the control devices, without compromising short-circuit current density $\left(J_{\mathrm{sc}}\right)$ and fill factor $(\mathrm{FF})$. The champion SSG-G device showed a PCE of $21.51 \%$ and a stabilized power output (SPO) of $20.91 \%$, as we show in Fig. 2B. We integrated the external quantum efficiency (EQE) spectra over the AM 1.5G solar spectrum, and the resulting $J_{\text {sc }}$ values were in close agreement with the values determined from the $J$ - $V$ scans (fig. S3). We fabricated 200 devices from different batches and present the histograms of average $V_{\text {oc }}$ values in Fig. $2 \mathrm{C}$ (we show histograms of the corresponding PCEs in fig. S4). The average $V_{\text {oc }}$ of the control devices was about $1.10 \mathrm{~V}$, whereas it was about $1.20 \mathrm{~V}$ for the SSG-G devices, with a record value of $1.21 \mathrm{~V}$ (Fig. 2C). The "stabilized photovoltage" values that we measured under continuous 1-sun illumination were consistent with the $V_{\text {oc }}$ values obtained from the scanned $J-V$ curves (Fig. 2D). In order to confirm our in-house device efficiency measurements, we sent one of our non-encapsulated devices to the National Institute of Metrology, China, for external certification and obtained a PCE of $20.90 \%\left(V_{\mathrm{oc}}=1.175 \mathrm{~V}, J_{\mathrm{sc}}=21.86 \mathrm{~mA} \mathrm{~cm} \mathrm{~cm}^{-2}\right.$, and FF $=$ $81.37 \%$ ) (fig. S5). A $V_{\mathrm{oc}}$ of $1.21 \mathrm{~V}$ rivals the values reported for the regular PSCs of similar bandgaps, corresponding to a "voltage deficit" of $0.41 \mathrm{~V}$. We compare our results to other published works for inverted PSCs in fig. S6 and table S7. The SSG-G process is also applicable to devices with varied electron-extraction layers, buffer layers, and metal electrodes (fig. S7 and table S8).

To assess the impact of SSG-G process on the long-term stability of the PSCs, we first tested the thermal stability of non-encapsulated devices in a nitrogen atmosphere. The SSG-G device showed a slight decay $(\sim 5 \%)$ in PCE after aging for $500 \mathrm{~h}$ at $85{ }^{\circ} \mathrm{C}$ (fig. S8). In contrast, the control device degraded more substantially to a PCE of $<60 \%$ of its original value, indicating that the SSG-G process improved the thermal stability of the device. We further examined the operational stability of non-encapsulated devices in a nitrogen atmosphere by comparing the PCE decay of the control and the SSG-G devices aging under Xenon-lamp-based simulator with an ultravoilet component $\left(100 \mathrm{~mW} \mathrm{~cm}^{-2}\right)$ at room temperature (Fig. 2B). The SPO of the control device showed a fast decay initially and then a subsequent decay at a relatively slow rate. In 40 contrast, we did not see an obvious decay in the SSG-G device over the measurement time. These results indicate that the positive impact caused by the SSG-G process is stable for an operational device that it improves the overall stability of the device.

We investigated the changes in the bandgap, microstrain, and electronic disorder induced by the SSG-G process (figs. S9 and S10) and observed some improvements, including a 45 reduction in the Urbach energy (described in the supplementary materials). However, by 
calculating the $V_{\mathrm{oc}}$ in the radiative limit (28), we confirmed that the reduced electronic disorder within the SSG-G film only contributed a few millivolts to the enhancement in $V_{\text {oc }}$ (fig. S11).

To uncover the origin of the $V_{\mathrm{oc}}$ rise, we performed surface photovoltage (SPV), ultraviolet photoelectron spectroscopy (UPS) and photoluminescence quantum yield (PLQY) measurements on a series of samples. We performed SPV measurements with a Kelvin probe on perovskite films coated on indium tin oxide (ITO) (structure 1), ITO/PTAA (structure 2) and on close-to-complete cells consisting of ITO/PTAA/perovskite/PC ${ }_{61} \mathrm{BM}$ (structure 3) (Fig. 3A). A $>100-\mathrm{meV}$ shift in surface potential for all films treated with the SSG-G process (compared with the control films) regardless of structure or illumination intensity. This shift was in the right direction to result in a $\sim 100-\mathrm{mV}$ increase in $V_{\text {oc }}$ in a cell.

We further investigated the electronic structures of the perovskite films by using UPS; we show the data for the control and the SSG-G perovskite films in Fig. 3B. From the secondary electron cut-off spectrum, we observed a change in work function from 5.02 to $4.42 \mathrm{eV}$ between the control and the SSG-G film. This is consistent with the direction of change, though slightly smaller in magnitude, that we determined from the SPV measurements. The valence band maximum $\left(E_{\mathrm{v}}\right)$ with respect to Fermi level $\left(E_{\mathrm{F}}\right)$ in the SSG-G film was also shifted relative to the control film (The absolute value of $E_{\mathrm{v}}$ can be calculated from the relationship shown in fig. S12). From these measurements, we built energy level diagrams for the two films (Fig. 3C). From the control to the SSG-G film, the $E_{\mathrm{v}}$ shifts by about $200 \mathrm{meV}$ toward vacuum, and the $E_{\mathrm{F}}$ shifts by a further $400 \mathrm{meV}$ toward the conduction band minimum $\left(E_{\mathrm{c}}\right)$. The $E_{\mathrm{F}}$ shift indicates a more n-type in nature for the SSG-G film, resulting from a surface and/or a bulk effect. The absolute shift in energy levels toward vacuum in the SSG-G film is likely to originate from a change in the ration of lead halide- to organic halide-terminated surfaces. Recently, lead iodide termination has been shown to lead to deeper energy levels than ammonium-iodide termination (29). Because we have added additional guanidinium bromide as a secondary process, we would expect a larger fraction of the surface to be terminated with organic cation halide.

Therefore, combining both the SPV and the UPS analysis, we obtained consistent results about a more n-type perovskite film produced by the SSG-G process, with the energy levels shifted toward vacuum. We characterized the uniformity of the SSG-G film by Kelvin probe force microscopy (KPFM) mapping (fig. S13) and found variation in surface potential similar to that of other reported conventional perovskite films (30).

To investigate the influence of the SSG-G process on the radiative recombination at the perovskite charge-extraction layer heterojunctions, we measured the PLQY for the films with various structures (Table 1). Isolated SSG-G films on quartz exhibited a higher PLQY of $\sim 2.8 \%$ compared with the control films at $\sim 0.17 \%$. However, on ITO substrates, which are the semitransparent electrodes used in devices, the SSG-G films exhibited PLQY similar to those of the control films. After the introduction of PTAA, the PLQY of the SSG-G films are 10 times those of the control films, reaching an average value of $8.9 \%$. The PLQY values were still $>2.5 \%$ when the PTAA and $\mathrm{PC}_{61} \mathrm{BM}$ layers simultaneously contact the SSG-G films, whereas the PLQY for the control film were quenched to an undetectably low level $(<0.1 \%)$. The radiative efficiencies of the perovskite films are influenced by the nature of the underlying substrate on which they are crystallized (31). Therefore, the factors that influence the PLQY are the "quality" of the ascrystallized perovskite films, the extent to which the substrate or subsequent layer extracts charges, and the degree to which new nonradiative recombination pathways are introduced at the perovskite contacting-layer heterojunctions. Evidently, ITO/PTAA is a good substrate on which 
to crystallize perovskite films; few nonradiative pathways appears to be introduced by the SSG$\mathrm{G}$ films contacting PTAA, and with sequent coating with $\mathrm{PC}_{61} \mathrm{BM}$, few additional nonradiative pathways are introduced. As it is evident from the high PCEs of the solar cells, these interfaces allow charge extraction.

To corroborate the PLQY results with the improved $V_{\mathrm{oc}}$ in the solar cells, we measured the electroluminescence from the PSCs. We observed an electroluminescence spectrum at 778 nanometers (nm) for the SSG-G device (fig. S14A), with an external radiative efficiency (ERE) of $1.2 \%$ under a forward bias current density of $23 \mathrm{~mA} \mathrm{~cm}^{-2}$, but we could not detect electroluminescence from the control device. The ERE value is lower than the PLQY of the perovskite films contacting both PTAA and $\mathrm{PC}_{61} \mathrm{BM}$. This is likely to be due to the isolated film emitting in both directions in the PLQY measurements, whereas the PSCs can only emit in the forward direction, implying more reabsorption of emitted light for the latter. Furthermore, there are likely imperfections in the deposition of the films, resulting in some shunting paths between the top metal electrode and the perovskite film, which could increase dark current and reduce ERE of the PSCs. We estimated a $V_{\text {oc }}$ of $1.21 \mathrm{~V}$ on the basis of the measured radiative efficiency (fig. S14B), which is in direct agreement with our device measurements under simulated 1-sun illumination.

To examine whether the electronic changes to the film are homogeneous through the thickness of the perovskite layer, or solely present in a surface region, we measured timeresolved photoluminescence (PL) spectra of perovskite films [coated on quartz substrates: quartz glass/perovskite/poly(methyl methacrylate) (PMMA)) and excited the film from glass and PMMA sides, using a 400-nm laser with 90-fs pulse width. We observed a single band emission of the control film when exciting the film from both PMMA and glass sides (Fig. 4A). In contrast, for the SSG-G film, we observed a dual-peak at an early time ( $0 \mathrm{~ns})$ when exciting the film from the top PMMA side, and this peak shifted from 735 to $767 \mathrm{~nm}$ over a few nanoseconds (Fig. 4C). However, when we excited the film from the glass side, we only observed the redshifted band $(\sim 767 \mathrm{~nm})$. We further performed transient absorption (TA) measurements by exciting the film with $400-\mathrm{nm}$ ( $90 \mathrm{fs}$ ) pump pulses and probing with broadband visible pulses. For the control film, we did not observe a considerable shift in the ground-state bleach upon exciting from either side (Fig. 4B). In contrast, again, for the SSG-G films, we observed a redshift of the ground state bleach-maxima from 730 to $750 \mathrm{~nm}$ over the first a few nanoseconds when exciting and probing from PMMA side (Fig. 4D), we did not observe such a redshift when exciting and probing from the glass side.

These time-resolved PL and TA results suggest the presence of a wider bandgap ( $~ 80$ meV wider) in the SSG-G film close to the surface. The resulting redshift in the time-resolved PL and TA spectra over time indicates that the charge population migrates from the wider bandgap region to the narrower bandgap bulk. Because we pumped the perovskite films with high energy photons $(400 \mathrm{~nm})$, we expect that the light was absorbed strongly in the top region (1/e absorption depth, $\sim 62 \mathrm{~nm}$ ), and the initial photoexcited charge population was hot. The time scale that we determined for the transfer of the charge population from the top region to the narrower bandgap bulk region is on the order of a few nanoseconds-much longer than the charge population cooling time, which is expected to occur within $100 \mathrm{fs}$ (32). Therefore, we expect that an equilibrium population of free carriers is created in the topmost region of the film, before charge transfer toward the bulk, most likely driven by diffusion. These results are consistent with establishing a wider bandgap close to the film surface. 
In EDX bromide elemental mapping (fig. S15) and time-of-flight secondary ions mass spectroscopy (ToF-SIMS) depth profiles (fig. S16), we observed a bromide-rich region close to the top surface of the SSG-G film. The Br enrichment near the top surface is consistent with a wider bandgap existing in this region of the film. Our studies of the spectroscopy and the stability of the PSCs suggest that the presence of the wider bandgap top layer is stable. Unexpectedly, we observed that the guanidinium $\left(\mathrm{GA}^{+}\right)$is well distributed across the perovskite layer. Because $\mathrm{GA}^{+}$is a large cation, and because in XRD measurements, we observed negligible shifts in characteristic perovskite reflection peaks, we would not expect a considerable fraction of $\mathrm{GA}^{+}$to be incorporated into the perovskite crystal lattice. Therefore, its location is most likely to at the surface and grain boundaries. A substantive contribution to the reduction in nonradiative recombination may therefore be through a passivation role(33), given that $\mathrm{GA}^{+}$may be present at both top and bottom surfaces and at the grain boundaries.

Multiple factors are thus responsible for the reduced nonradiative recombination. First, the predominant trap-species leading to trap-assisted recombination in perovskite films are electron traps (34). The more n-type perovskite film could result in a larger fraction of occupied versus vacant traps and a reduction in the rate of trap-assisted recombination. Secondly, a wider bandgap near the top surface of the SSG-G film might reduce the total electron-hole recombination rate in this region of the film where surface defects are likely to exist, by making it energetically favorable for one or both charge carriers to reside predominantly within the bulk of the film, away from the surface. Thirdly, the presence of guanidinium halide throughout the films and at the surfaces may inhibit unwanted trap-assisted recombination at the heterojunctions by trap passivation. Hence, the SSG-G process that we have presented to improve the perovskite film quality results in considerable improvements to the electronic nature at the heterojunctions between the perovskite film and charge-extraction layers. This should be broadly applicable to PSCs and perovskite light emitting diodes.

\section{REFERNCES AND NOTES}

1. K. A. Bush et al., Nat. Energy 2, 17009 (2017).

2. J. Zhao et al., Energy Environ. Sci. 9, 3650-3656 (2016).

3. X. Zheng et al., Nat. Energy 2, 17102 (2017).

4. W. Chen et al., Science 350, 944-948 (2015).

5. M. Saliba et al., Science 354, 206-209 (2016).

6. L. Ling et al., Adv. Funct. Mater. 26, 5028-5034 (2016).

7. J. Huang, Y. Yuan, Y. Shao, Y. Yan, Nat. Rev. Mater. 2, 17042 (2017).

8. S. Chen et al., Adv. Energy Mater. 6, 1600132 (2016).

9. W. Zhang, G. E. Eperon, H. J. Snaith, Nat. Energy 1, 16048 (2016).

10. D. W. deQuilettes et al., Science 348, 683-686 (2015).

11. G. Han et al., ACS Appl. Mater. Interfaces 9, 21292-21297 (2017).

12. K. T. Cho et al., Energy Environ. Sci. 10, 621-627 (2017).

13. N. De Marco et al., Nano Lett. 16, 1009-1016 (2016).

14. D. Bi et al., Sci. Adv. 2, e1501170 (2016).

15. D. Shi et al., Science 347, 519-522 (2015).

16. F. Deschler et al., J. Phys. Chem. Lett. 5, 1421-1426 (2014).

17. G. E. Eperon, D. Moerman, D. S. Ginger, ACS Nano 10, 10258-10266 (2016).

18. M. Yang et al., Nat. Commun. 7, 12305 (2016).

19. W. Nie et al., Science 347, 522-525 (2015). 
20. Y. Shao, Y. Yuan, J. Huang, Nat. Energy 1, 15001 (2016).

21. Y. Wu et al., Nat. Energy 1, 16148 (2016).

22. M. Liu, M. B. Johnston, H. J. Snaith, Nature 501, 395-398 (2013).

23. T. Leijtens et al., ACS Nano 8, 7147-7155 (2014).

24. X. Zhang et al., J. Phys. Chem. Lett. 7, 4602-4610 (2016).

25. P. Schulz et al., Adv. Mater. Interfaces 2, 1400532 (2015).

26. Y. C. Kim et al., Adv. Energy Mater. 6, 1502104 (2016).

27. Q. Chen et al., Nano Lett. 14, 4158-4163 (2014).

28. K. Tvingstedt et al., Sci. Rep. 4, 6071 (2014).

29. C. Quarti, F. D. Angelis, D. Beljonne, Chem. Mater. 29, 958-968 (2017).

30. J. J. Li et al., ACS Appl. Mater. Interfaces 7, 28518-28523 (2015).

31. C. Bi et al., Nat. Commun., 6, 7747 (2015).

32. M. B. Price et al., Nat. Commun. 6, 8420 (2015).

33. X. Hou et al., J. Mater. Chem. A 5, 73-78 (2017).

34. S. D. Stranks et al., Phys. Rev. Applied 2, 034007 (2014).

ACKNOWLEDGMENT: We thank S. Hinder from Faculty of Engineering and Physical Sciences, University of Surrey (U. K.) for his kind assistance in ToF-SIMS measurement and helpful discussion, B. Wenger from University of Oxford (U. K.) for his assistance in PLQY measurement, S. Mahesh from University of Oxford (U. K.) for his help with radiative loss estimation, P.Li and Z. Lu from Department of Materials Science and Engineering at University of Toronto (Canada), for helpful suggestions on UPS measurement and analysis. Funding: This work was partly funded by the 973 Program of China (2015CB932203), the National Natural Science Foundation of China (61722501, 91733301, 91433203 and 61377025), EPSRC (UK), the European Union Seventh Framework Programme under grant agreement 604032 of the MESO project, and AFOSR through project FA9550-15-1-0115. W.Z. acknowledges financial support from a Royal Society Research Grant (2017; RG160742), the Royal Society International Exchanges Scheme (2016; IE160511), and the University of Surrey Sustainability Conference Support Programme. A.S. and R.H.F. acknowledge support from EPSRC, Indo-UK APEX, and UKIERI projects. R. Sh. acknowledges Newton-Bhabha international fellowship. Authors contributions: D.L. and R.Z. conceived of the work. D.L. and W.Y. fabricated and characterized solar cells. D.L. and W.Y. conducted UPS, KPFM, electroluminescence, ERE, and EQE measurements. Z.W. conducted SPV measurements and estimated microstrain and the voltage radiative limit. Z.W. and R.Sh. conducted PLQY measurements. A.S. and R.H.F. contributed to the photothermal deflection spectroscopy data. A.S., R.Sh., and R.H.F contributed to time-resolved PL and TA data. Q.G. analyzed and discussed time-resolved PL and TA spectra. Q.H. conducted SEM experiments on the perovskite films, performed SEM analysis, and conducted the XRD measurements. L.Z. and F.Y. analyzed the XRD data. R.Su., T.L., K.C., and D.L. contributed to the certification of solar cells. R.Su. and P.W. conducted thermal stability tests on the devices. Y.T. and J.W. conducted EDX measurements, and L.Z. contributed to the EDX analysis. Y.Z. prepared metal oxide buffer layers. Y.Z. and X.Y. measured ultravioletvisible light absorption spectra. G.F.T., J.F.W., R.Su., D.L., and W.Z. contributed to the ToF- 
SIMS measurement and data analysis. Z.X. conducted statistical analysis of device efficiencies. D.L. and Z.W. estimated the voltage deficit from bandgap to voltage. W.Z., H.J.S., and R.Z. directed and supervised the project. D.L. and W.Y. wrote the first draft of the paper. Z.W., W.Z., H.J.S., and R.Z. revised the paper. All authors analyzed their data and reviewed and commented on the paper. Competing interests: H.J.S. is chief scientific officer of Oxford PV, a company commercializing perovskite solar cells. Data and materials availability: All data needed to evaluate the conclusions in the paper are present in the paper or the supplementary materials.

\section{SUPPLEMENTARY MATERIALS}

Materials and Methods

10 Supplementary Text

Figs. S1 to S16

Tables S1 to S8

References (35-48) 

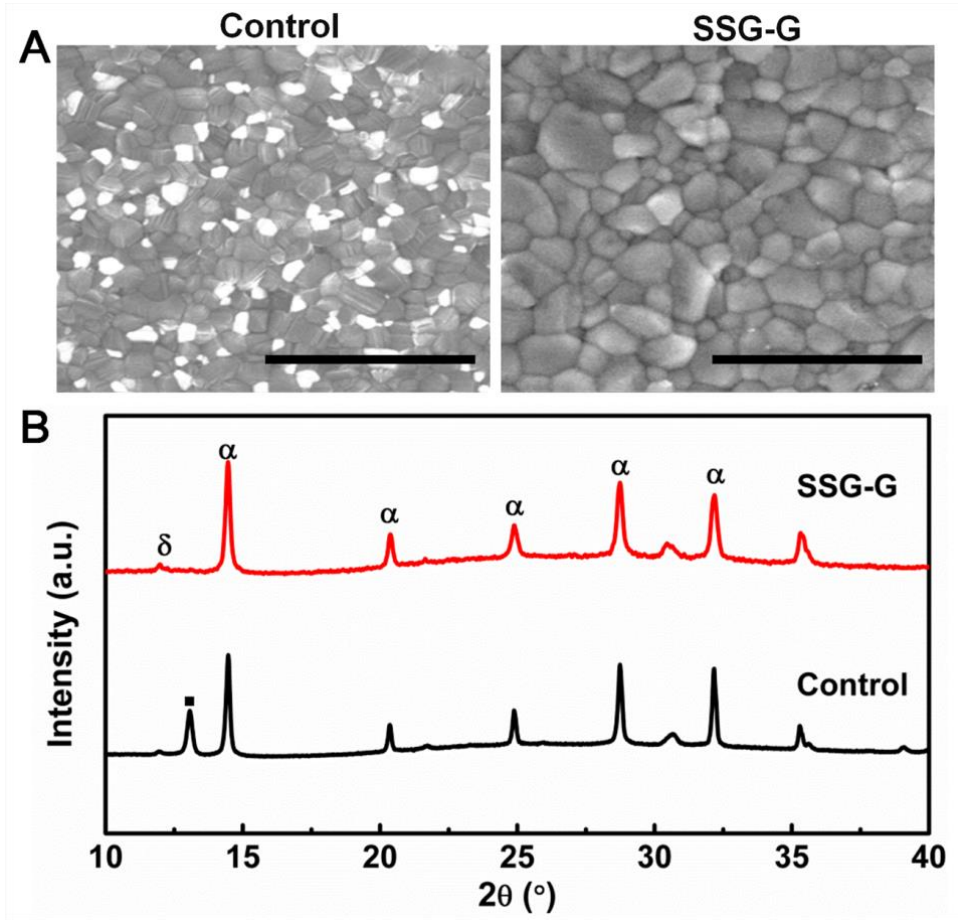

Fig. 1. Improved morphologies and crystal structures. (A) Top-view SEM images of the control and SSG-G films. Scale bar, $2 \mu \mathrm{m}$. (B) XRD patterns $(\alpha, \delta$ and black square denote the identified diffraction peaks corresponding to black-perovskite phase, non-perovskite phase and $\mathrm{PbI}_{1.50} \mathrm{Br}_{0.50}$, respectively). a.u., arbitrary units. 

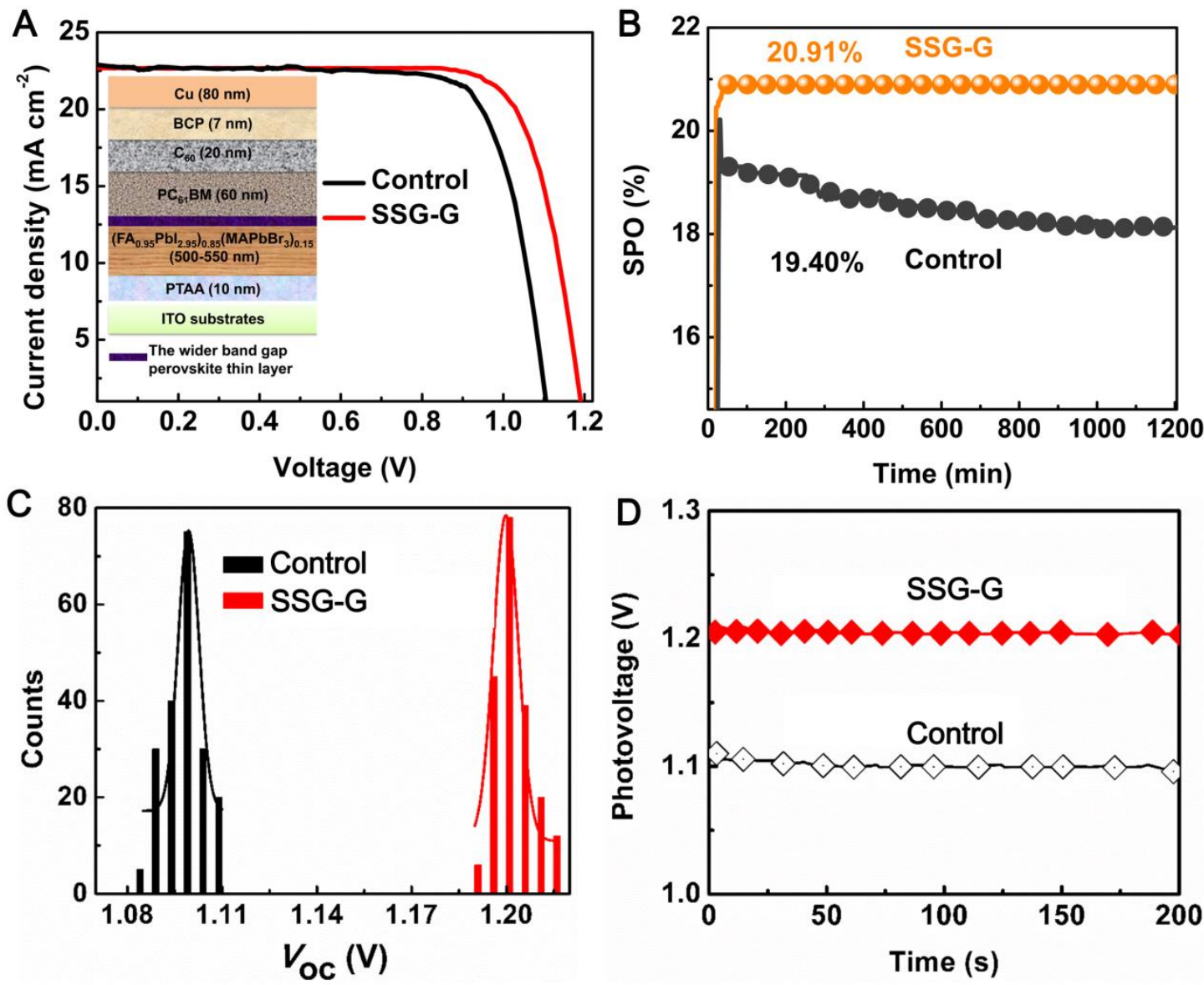

Fig. 2. Photovoltaic performances. (A) The $J$ - $V$ curves of the inverted planar heterojunction PSCs obtained from reverse scans under simulated AM $1.5 \mathrm{G}$ illumination at $100 \mathrm{~mW} \mathrm{~cm}$. The inset shows a schematic diagram of the solar cell used in this work. (B) The stabilized power output (SPO) for champion device and the control device. The SPO at the maximum power point is indicated. (C) Histograms of the $V_{\mathrm{oc}}$ for 200 control and 200 the SSG-G devices. (D) Stabilized photovoltage as a function of illumination time at the open-circuit conditions for the champion device and the control device. 

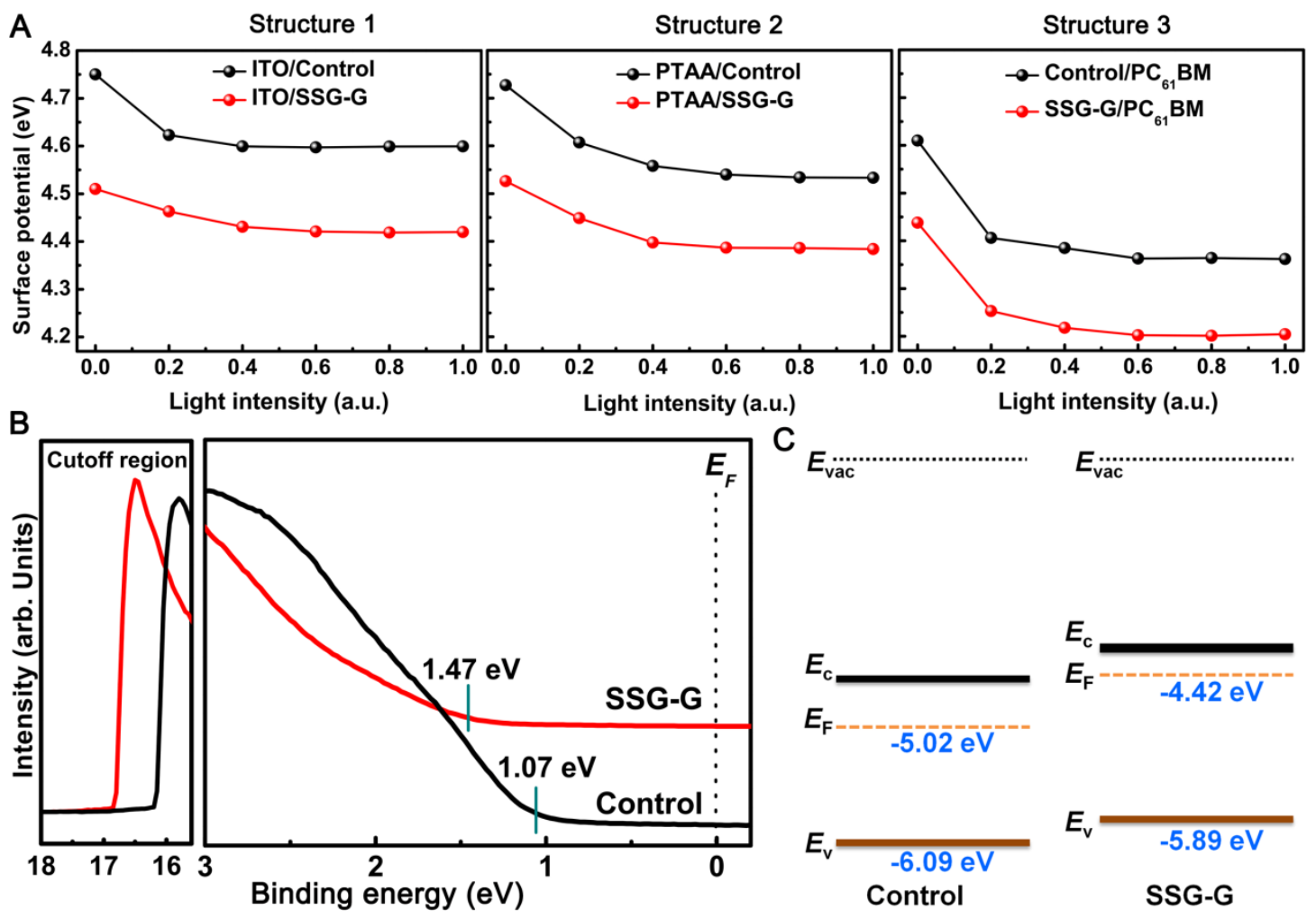

Fig. 3. Surface photovoltage and ultraviolet photoelectron spectroscopy. (A) Surface photovoltage measurements of the perovskite films coated on ITO (structure 1), ITO/PTAA (structure 2) and on close-to -omplete cells consisting of ITO/PTAA/perovskite/ $\mathrm{PC}_{61} \mathrm{BM}$ (structure 3). (B) Helium I $\alpha(h v=21.22 \mathrm{eV})$ spectra of secondary electron cut-off and valence band of the control and SSG-G perovskite thin films. The blue-green vertical lines indicate the valence band maximum $\left(E_{\mathrm{v}}\right)$ with respect to the Fermi level. (C) Schematic energy-level diagrams of control and SSG-G films. 

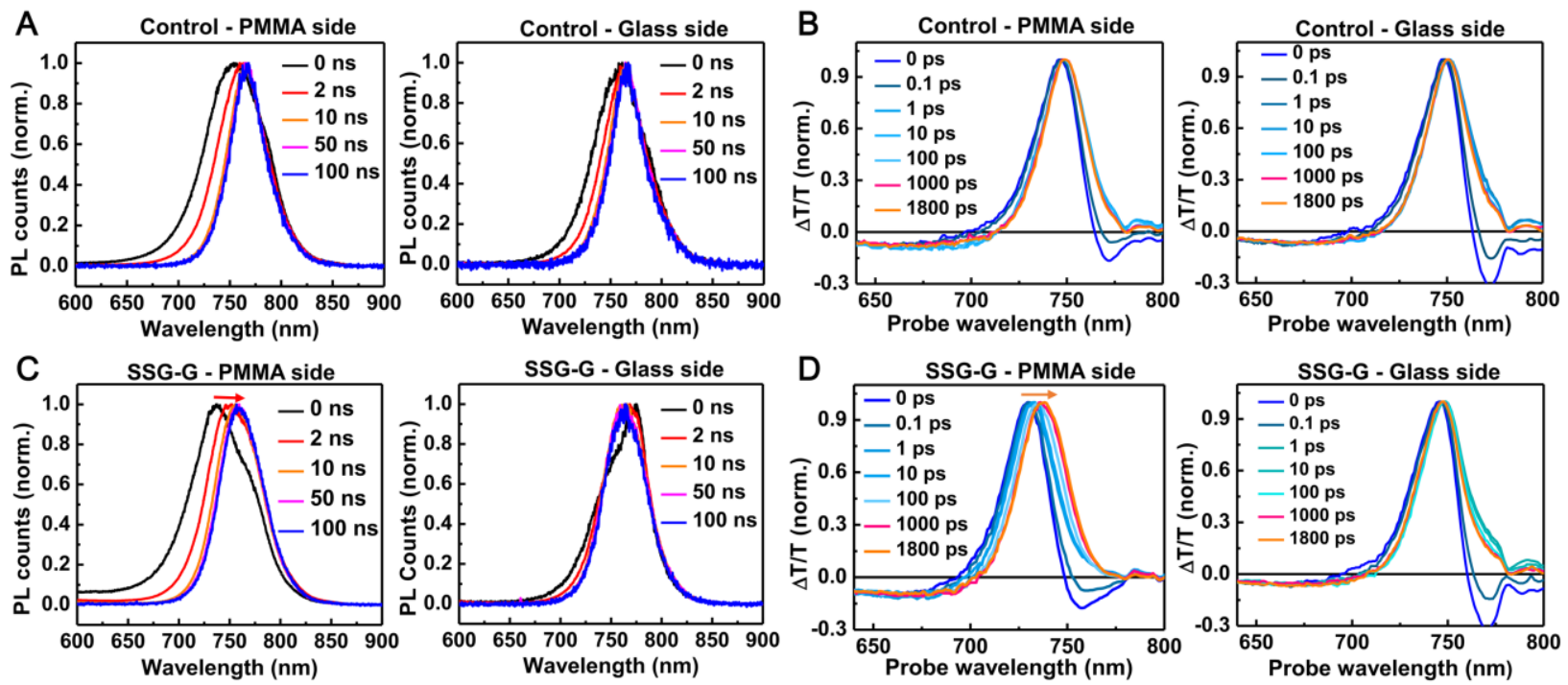

Fig. 4. Time-resolved photoluminescence and transient absorption spectra. Photoluminescence spectra (A and $\mathbf{C}$ ) of the films consisting of quartz glass/perovskite/PMMA, at different time scales after excitation, recorded from both PMMA and glass sides in the reflection geometry, for (A) control and (C) SSG-G samples. (B and D) Transient absorption spectra of films consisting of the quartz glass/perovskite/PMMA in the transmission geometry, obtained by using 400-nm (90-fs) excitation pump pulse from different sides, for (B) control and (D) SSG-G samples.

Table 1. Photoluminescence quantum yield (PLQY). Summary of PLQY results for the control and SSG-G perovskite films on the quartz, ITO, ITO/PTAA, and on close-to-complete cells consisting of ITO/PTAA/perovskite/PC ${ }_{61} \mathrm{BM}$.

\begin{tabular}{lll} 
Substrates & Samples & PLQY $(\%)$ \\
\hline Quartz & Control & $0.172 \pm 0.001$ \\
Quartz & SSG-G & $2.843 \pm 0.002$ \\
ITO & Control & $1.704 \pm 0.096$ \\
ITO & SSG-G & $1.499 \pm 0.384$ \\
ITO/PTAA & Control & $0.858 \pm 0.368$ \\
ITO/PTAA & SSG-G & $8.908 \pm 0.643$ \\
ITO/PTAA & Control/PC ${ }_{61} \mathrm{BM}$ & Below detection limit \\
ITO/PTAA & SSG-G/PC 61 BM & $2.506 \pm 0.599$ \\
\hline
\end{tabular}




\section{Science $\triangle \mathrm{TAAAS}$}

\section{Supplementary Materials for}

\section{Enhanced photovoltage for inverted planar heterojunction perovskite solar cells}

Deying Luo,* Wenqiang Yang,* Zhiping Wang,* Aditya Sadhanala, Qin Hu, Rui Su,

10 Ravichandran Shivanna, Gustavo F. Trindade, John F. Watts, Zhaojian Xu, Tanghao Liu, Ke Chen, Fengjun Ye, Pan Wu, Lichen Zhao, Jiang Wu, Yongguang Tu, Yifei Zhang, Xiaoyu Yang, Wei Zhang, $\uparrow$ Richard H. Friend, Qihuang Gong, Henry J. Snaith $\uparrow$, Rui Zhu†

*These authors contributed equally to this work

15 †Corresponding author: E-mail: wz0003@surrey.ac.uk (W.Z.); henry.snaith@physics.ox.ac.uk (H.J.S); iamzhurui@pku.edu.cn (R.Z.).

\section{This PDF file includes:}

Materials and Methods

Supplementary Text

Figs. S1 to S16

Tables S1 to S8

25

References 


\section{Materials and Methods}

Materials and solvents

Methylamium bromide (MABr) and formamidinium iodide (FAI) were synthesized using the methods reported by the previous literatures (35-37). Guanidinium iodide (GAI, >98.0\%), guanidinium bromide $(\mathrm{GABr},>98.0 \%)$ and guanidinium chloride $(\mathrm{GACl},>98.0 \%)$, and formamidinium chloride ( $\mathrm{FACl}, 99.8 \%$ ) were purchased from Tokyo Chemical Industry Co., Ltd. (TCI, Japan). Lead diiodide $\left(\mathrm{PbI}_{2}, 99.99 \%\right)$ and lead dibromide $\left(\mathrm{PbBr}_{2}, 99 \%\right)$ were also purchased from the TCI (Japan). Formamidinium bromide (FABr, >99\%), and poly[bis (4phenyl)(2,4,6-trimethylphenyl)amine] (PTAA, $\mathrm{Mn}=3200, \mathrm{Mw}=4900$ ) were purchased from Xi'an Polymer Light Technology Corp (China), and [6,6]-phenyl-C61-butyric acid methyl ester $\left(\mathrm{PC}_{61} \mathrm{BM}\right)$ was purchased from Nano-C Tech. (USA). 2,3,5,6-Tetrafluoro-7,7,8,8tetracyanoquinodimethane $\left(\mathrm{F}_{4}\right.$-TCNQ, 99\%), bathocuproine (BCP, 99.9\%), and buckminsterfullerene $\left(\mathrm{C}_{60}, 99 \%\right)$ were purchased from Jilin OLED Company (China). 3,9-bis(2methylene-(3-(1,1-dicyanomethylene)indanone)-5,5,11,11-tetrakis(4-hexylphenyl)dithieno[2,3$\mathrm{d}: 2$ ',3'-d']-s-indaceno[1,2-b:5,6-b']dithiophene) (ITIC) was received from Borun New Material Company (China). Indium tin oxide (ITO) nanoparticle dispersion in isopropanol was purchased from the Sigma Aldrich (USA). Poly(methyl methacrylate) (PMMA) received from commercial source. Besides, some liquid reagents, including $N, N$-dimethylformamide (DMF, 99.8\%), dimethyl sulfoxide (DMSO, 99.7\%), isopropanol (IPA, 99.5\%) and chlorobenzene (CB, purity of 99.8\%) were purchased from commercial sources (Acros) and used as received. Toluene was purchased from Sinopharm Chemical Reagent Co., Ltd. All metal materials including gold (Au), silver $(\mathrm{Ag})$, copper $(\mathrm{Cu})$, and chromium $(\mathrm{Cr})$ were obtained from commercial sources with high purity $(\geq 99.99 \%)$.

$\underline{\text { Perovskite precursor solution }}$

The perovskite precursor solution was prepared by mixing FAI (1.09 $\mathrm{M}), \mathrm{PbI}_{2}(1.15 \mathrm{M})$, $\operatorname{MABr}(0.20 \mathrm{M})$ and $\operatorname{PbBr}_{2}(0.20 \mathrm{M})$ in a mixed solvent of DMF/DMSO (4/1, v/v). The prepared solution was stirred at $70-80{ }^{\circ} \mathrm{C}$ for $15 \mathrm{~min}$ before use. For the desirable secondary growth solution, FAX (X = I and $\mathrm{Br})$ and/or $\mathrm{GAX}(\mathrm{X}=\mathrm{I}$ and $\mathrm{Br})$ were dissolved into IPA solvent respectively, and the concentrations ranged from 3 to $6 \mathrm{mg} \mathrm{mL}^{-1}$.

\section{Doped-PTAA and $\mathrm{PC}_{61}$ BM solutions}

We first made a stock solution of $\mathrm{F}_{4}$-TCNQ in $\mathrm{CB}\left(1 \mathrm{mg} \mathrm{ml}^{-1}\right)$, which was stirred at $60{ }^{\circ} \mathrm{C}$ for $12 \mathrm{~h}$. $\mathrm{F}_{4}$-TCNQ solution was then added into PTAA solution $\left(10 \mathrm{mg} \mathrm{ml}^{-1}\right.$ in toluene) with a weight ratio of $1 \%$. $\mathrm{PC}_{61} \mathrm{BM}$ solution was prepared by dissolving $20 \mathrm{mg}$ of $\mathrm{PC}_{61} \mathrm{BM}$ into $\mathrm{CB}$ (1 $\mathrm{ml}$ ) and stirring at $70^{\circ} \mathrm{C}$ for $2 \mathrm{~h}$.

\section{$\underline{\text { Hole-extraction layer }}$}

The pre-patterned ITO substrates were ultrasonically cleaned with diluted detergent, deionized water, acetone, and isopropanol (IPA) in succession for $20 \mathrm{~min}$. The as-cleaned ITO substrates were treated by UV-ozone for $10 \mathrm{~min}$. The substrate's size is $1.5 \times 1.5 \mathrm{~cm}^{2}$. Subsequently, the ITO substrates were transferred to a $\mathrm{N}_{2}$-filled glovebox with $\mathrm{H}_{2} \mathrm{O}$ and $\mathrm{O}_{2}$ concentrations of $<0.1 \mathrm{ppm}$. $\mathrm{F}_{4}$-TCNQ-doped PTAA solution was spin-coated onto the ITO substrates at $4000 \mathrm{rpm}$ (with a ramping rate of $1000 \mathrm{rpm} \mathrm{s}^{-1}$ ) for $30 \mathrm{~s}$, and then the samples were heated at $150{ }^{\circ} \mathrm{C}$ for $10 \mathrm{~min}$ in a $\mathrm{N}_{2}$-filled inert atmosphere. To improve the wettability of 
perovskite precursor solution on the PTAA hole-extraction layer, the PTAA-coated ITO substrates were pre-treated by spin-coating $50 \mu \mathrm{l}$ of DMF at a speed of $5000 \mathrm{rpm}$ (with a ramping rate of $3000 \mathrm{rpm} \mathrm{s}^{-1}$ ) for $30 \mathrm{~s}$ prior to perovskite films deposition.

Perovskite films deposition

The mixed-cation lead mixed-halide perovskite solution (with or without $5 \mathrm{~mol} \%$ excess $\mathrm{PbI}_{2}$ ) was spin-coated on the PTAA-coated ITO substrates by a two-consecutive step program at $2000 \mathrm{rpm}$ for $10 \mathrm{~s}$ with a ramping rate of $200 \mathrm{rpm} \mathrm{s}^{-1}$, and $6000 \mathrm{rpm}$ for $30 \mathrm{~s}$ with a ramping rate of $1000 \mathrm{rpm} \mathrm{s}^{-1}$. During the second step, $100 \mu \mathrm{L}$ of anhydrous CB was poured on the center of the spinning substrates $16 \mathrm{~s}$ prior to the end of the whole spinning program. The samples were immediately annealed on a hotplate at $105{ }^{\circ} \mathrm{C}$ for $90 \mathrm{~min}$ in an inert atmosphere $\left(\mathrm{N}_{2}\right)$. To realize the solution-processed secondary growth, the films were cooled to room temperature and then were further grown with FAX (X = I and $\mathrm{Br}$ ) or GAX (X = I and Br) solution in IPA (which we abbreviate to SSG-F and SSG-G), respectively. Briefly, $100 \mu \mathrm{L}$ of FAX (X = I and Br)/IPA or GAX (X = I and Br)/IPA solution (the concentration ranged from 2 to $6 \mathrm{mg} \mathrm{mL}^{-1}$ ) was spincoated onto the as-prepared perovskite films at the speed of $5000 \mathrm{rpm}$ (with a ramping rate of $2500 \mathrm{rpm} \mathrm{s}^{-1}$ ) for $30 \mathrm{~s}$. The films were then heated at $105{ }^{\circ} \mathrm{C}$ for $10 \mathrm{~min}$. Note that FAX/IPA or GAX/IPA solution should be added onto the perovskite films very fast, and the solution needs to be kept on the films for $1-2 \mathrm{~s}$ before spin-coating. The temperature in the glovebox needs to be sustained at $\sim 25^{\circ} \mathrm{C}$. All steps were finished in a $\mathrm{N}_{2}$-filled glovebox, and some solvent vapor including DMF, DMSO, $\mathrm{CB}$, and toluene in the glovebox should be minimized during the perovskite films deposition process.

\section{Electron-extraction layer and metal electrodes}

To make an electron-extraction layer, $35 \mu \mathrm{L}$ of $\mathrm{PC}_{61} \mathrm{BM}\left(20 \mathrm{mg} \mathrm{mL}^{-1}\right.$ in $\left.\mathrm{CB}\right)$ solution was spin-coated on the top of perovskite layer at the speed of $1000 \mathrm{rpm}$ (with a ramping rate of 200 $\mathrm{rmp} \mathrm{s}^{-1}$ ) for $30 \mathrm{~s}$ in a $\mathrm{N}_{2}$-filled glovebox. Then, the samples were transferred to a vacuum chamber without being exposed to air. $\mathrm{C}_{60}(20 \mathrm{~nm}) / \mathrm{BCP}(7 \mathrm{~nm})$ were then thermally evaporated in a vacuum chamber with the base pressure of $<7 \times 10^{-5} \mathrm{~Pa}$. For comparison, an alternative electron-extraction layer was obtained by spin-coating a CB solution (ITIC, $4 \mathrm{mg} \mathrm{ml}^{-1}$ ) at a speed of $4000 \mathrm{rpm}$ for $20 \mathrm{~s}$. Similarly, both ITO nanoparticle and $\mathrm{CrO}_{\mathrm{x}}$ as alternative buffer layers to $\mathrm{BCP}$ were studied. The ITO nanoparticle buffer layer was prepared by spin-coating a commercial ITO nanoparticle dispersion with varied concentrations at $3500 \mathrm{rpm}$ for $20 \mathrm{~s}$. The $\mathrm{CrO}_{\mathrm{x}}$ buffer layer was obtained through the oxidation of metal $\mathrm{Cr}(1.5 \mathrm{~nm})$ in an atmosphere containing oxygen. Subsequently, the metal electrode, such as $\mathrm{Au}, \mathrm{Ag}$, and $\mathrm{Cu}$, was thermally evaporated in a separate vacuum chamber $\left(<4 \times 10^{-4} \mathrm{~Pa}\right)$ through a metal shadow mask with an aperture area of $0.09 \mathrm{~cm}^{2}$. The thickness of metal electrode was $80 \mathrm{~nm}$, and the thermal evaporation rate ranged from 0.5 to $2 \AA \mathrm{A} \mathrm{s}^{-1}$ depending on the metal used.

$40 \quad$ Solar cell characterization

The current density-voltage $(J-V)$ curves of solar cells were measured (2400 Series SourceMeter, Keithley Instruments) under simulated AM $1.5 \mathrm{G}$ sunlight at $100 \mathrm{~mW} \cdot \mathrm{cm}^{-2}$ irradiance by a $150 \mathrm{~W}$ class AAA solar simulator (XES-40S1, SAN-EI). The light intensity of $100 \mathrm{~mW} \cdot \mathrm{cm}^{-2}$ was calibrated by using a standard monocrystalline silicon solar cell with a KG-5 45 filter. To ensure the accuracy of the $J_{\text {sc }}$ measured from $J$ - $V$ scans, a mask with an aperture area of $0.07 \mathrm{~cm}^{2}$ was employed during the measuring process. The sweeping conditions are: reverse scan 
$\left(1.22 \mathrm{~V} \rightarrow-0.02 \mathrm{~V}\right.$, scan rate $40 \mathrm{mV} \mathrm{s}^{-1}$, and no delay time) and forward scan $(-0.02 \mathrm{~V} \rightarrow 1.22 \mathrm{~V}$, scan rate $40 \mathrm{mV} \mathrm{s}^{-1}$, and no delay time). The $J-V$ measurements of non-encapsulated solar cells were performed in a $\mathrm{N}_{2}$-filled glovebox. Stabilized power output (SPO) and stabilized photovoltage were also measured in a $\mathrm{N}_{2}$-filled glovebox under simulated AM 1.5G, $100 \mathrm{~mW}$ $\mathrm{cm}^{-2}$ solar irradiation by a $150 \mathrm{~W}$ class AAA solar simulator with an AM 1.5G filter, which was recorded with an electrochemical workstation (Autolab PGSTAT302N, Metrohm, Switzerland).

\section{Thermal-stability test}

The stability of the non-encapsulated solar cell was tested in a nitrogen atmosphere, by tracking the device performance at $85^{\circ} \mathrm{C}$ for 500 hours.

\section{External quantum efficiency (EQE)}

The EQEs of the non-encapsulated solar cells were measured in air by a lock-in amplifier coupled with a monochromator (Crowntech, Qtest Station 2000 USA). A standardmonocrystalline silicon cell was used as the reference for the EQE tests. No pre-light soaking and pre-bias were applied over the measurement time.

\section{Electroluminescence and external radiative efficiency (ERE)}

Electroluminescence and external radiative efficiency measurements were performed by applying different voltages to the device with a digital source meter (Keithley 2400). The device was placed on a 100-mm-diameter integrating sphere (NBeT Group Corp.), and the emitted photon flux was recorded by spectrometer (OceanOptics HR4000). The system was calibrated by a photodiode.

Scanning electron microscopy (SEM) and energy dispersive X-ray spectroscopy (EDX)

The surface morphologies and element distributions of perovskite films coated on ITO substrates without (control) and with SSG process were studied by SEM (FEI Nova_Nano SEM 430, $10 \mathrm{KV}$ ) and EDX analysis (20 KV, Thermo Scientific Noran System 7). The perovskite films were made by the same protocols used in solar cell fabrication.

Ultraviolet-visible (UV-Vis) absorption spectra

The samples for UV-Vis characterization were made by depositing perovskite films on the PTAA-coated ITO substrates. UV-Vis absorption nature $(300-850 \mathrm{~nm})$ was then recorded with a spectrophotometer (UH4150, Hitachi, Japan). During the measuring process, the perovskite thin films were exposed to air less than $5 \mathrm{~min}$, and the background noise was subtracted before testing.

$\underline{\text { X-ray diffraction (XRD) characterization }}$

The samples were made by depositing perovskite films on the ITO substrates. XRD patterns 40 were performed using $40 \mathrm{kV}, 40 \mathrm{~mA} \mathrm{Cu} \mathrm{K \alpha}(\lambda=0.15406 \mathrm{~nm})$ radiation by Mini Flex 600 (Rigaku, Japan).

\section{Photothermal deflection spectroscopy (PDS)}

For the PDS measurements, perovskite thin films were prepared on quartz glasses to 
protection layer was deposited onto the perovskite to prevent damaging perovskite films caused by moisture and other environmental factors. The PMMA protection layer was made by spincoating a CB solution $\left(10 \mathrm{mg} \mathrm{mL}^{-1}\right.$ ) at a speed of $3000 \mathrm{rpm}$ (with a ramping rate of $1500 \mathrm{rpm} \mathrm{s}^{-1}$ ) for $30 \mathrm{~s}$. During the measurement, the samples were kept in a hermetically sealed quartz cuvette filled with an inert liquid, Fluorinert FC-72 from 3M Corporation, which acts as deflection medium with high-temperature-dependent refractive index. The perovskite films were excited from the quartz side with a modulated monochromatic light beam perpendicular to the plane of the sample. Modulated monochromatic light beam was produced by a combination of a light source (Light Support MKII, $100 \mathrm{~W}$ Xenon) and a monochromator (CVI DK240). The transverse probe beam was obtained with 670-nm fiber-coupled diode laser (Qioptiq) and passed as close as possible to the perovskite-film surface. Beam deflection was measured using a differentially amplified quadrant photodiode and a standard lock-in amplifier (SR830).

\section{Surface photovoltage (SPV) measurements by a Kelvin Probe}

Vibrating Kelvin Probe (probe diameter $=2 \mathrm{~mm}$ ) (KP Technology, UK) was used to determine the surface potential or the surface work function. The perovskite films were prepared on ITO (structure 1), ITO/PTAA (structure 2) and close to complete cells comprising of ITO/PTAA/perovskite/PC ${ }_{61} \mathrm{BM}$ (structure 3). The thickness of the perovskite film was around 500 - $550 \mathrm{~nm}$. Surface photovoltage (SPV) measurements in ambient were done under illumination from a 150-W quartz tungsten halogen lamp, where the intensity was controlled linearly by changing the power supplied to the lamp. Using an optical fiber and lens, light beam could fall on the perovskite surface at a 45-degree angle to illuminate the whole surface area. All measurements were done on different spots and different samples, and ultimately the average value is reported in this work.

\section{Ultraviolet photoelectron spectroscopy (UPS)}

The samples were coated on the highly conductive and low-surface roughness ITO substrates. The thicknesses of the films were about $100-150 \mathrm{~nm}$ to ensure good charge transporting at the surface of the perovskite films. UPS spectra were recorded with an Imaging Photoelectron Spectrometer (Axis Ultra, Kratos Analytical Ltd), with a non-monochromated He I $\alpha$ photon source $(h v=21.22 \mathrm{eV})$. The measurements were carried out with the samples tilted to a take-off angle of $90^{\circ}$ in an ultrahigh vacuum chamber with a base pressure of $\sim 10^{-10}$ torr.

\section{Kelvin probe force microscopy (KPFM)}

35 The KPFM measurements were carried out in room temperature and dark conditions (Dimension ICON, Bruker). The frequency-modulation KPFM (FM-KPFM) mode was used to probe top-view surface potential. The perovskite films used for the KPFM characterizations were prepared on the ITO substrates, and the process for making films was exactly the same as the device fabrication.

Photoluminescence quantum yield (PLQY)

The PLQY of the control and SSG-G processed perovskite samples were measured by an integration sphere. A continuous wave diode laser of $532 \mathrm{~nm}$ wavelength $\left(40 \mathrm{~mW} \mathrm{~cm}{ }^{-2}\right.$, which equates to approximately $100 \mathrm{~mW} \mathrm{~cm}^{-2} \mathrm{AM} 1.5 \mathrm{G}$ absorbed photon flux)) was used to photo45 excite the samples. The emission signal was collected using calibrated Andor iDus Si Detector and calculated using the method as described elsewhere (38). 
Time-resolved photoluminescence (PL) spectroscopy

For the time-resolved PL measurements, perovskite thin films were prepared on quartz glasses to minimize the light absorption of the substrates. Before the preparation of perovskite films, the quartz-glass substrates were treated with UV-ozone for $10 \mathrm{~min}$. The perovskite films were then prepared with the SSG process, and $40 \mu \mathrm{L}$ of PMMA solution $\left(10 \mathrm{mg} \mathrm{ml}^{-1}\right.$, in CB) was spun onto the perovskite at $3000 \mathrm{rpm}$ (with a ramping rate of $1500 \mathrm{rpm} \mathrm{s}^{-1}$ ) for $30 \mathrm{~s}$. The film architectures (quartz glass/perovskite/PMMA) were employed for time-resolved PL spectroscopy measurements. The time-resolved PL spectra were acquired using an electrically-gated intensified CCD (ICCD) camera (Andor iStar DH740 CCI-010) coupled to a calibrated grating spectrometer (Andor SR303i). The photoexcitation pulses (pulse wavelength $=400 \mathrm{~nm}$, pulse width $\approx 90 \mathrm{fs}$ ) are generated from the fundamental output pulses (pulse wavelength $=800 \mathrm{~nm}$, pulse width $=90 \mathrm{fs}$ and repetition rate $=1 \mathrm{kHz}$ ) of a Ti: Sapphire laser system (Spectra Physics Solstice) passing through the second harmonic generation (SHG) beta barium borate (BBO) crystal. The excitation and collection of PL spectra were from the same side of the sample in the reflection geometry. A $420 \mathrm{~nm}$ long-pass filter was placed before the spectrometer to prevent the laser scattering. Temporal evolution of the PL emission was acquired by varying electronic gate delay of ICCD camera with respect to the excitation pulse. The instrument resolution of electronic ICCD gate step was $~ 2 \mathrm{~ns}$.

Transient absorption (TA) spectroscopy

The samples (film architectures: quartz glass/perovskite/PMMA) used for time-resolved PL measurements were further used to record transient absorption spectroscopy. The fundamental output of a Ti: Sapphire amplifier system (Spectra-Physics Solstice) operating at $1 \mathrm{KHz}$ (Pulse wavelength $=800 \mathrm{~nm}$, pulse width $=90 \mathrm{fs}$ ) was split into two beams. One beam pulse was converted into the broadband visible probe by the home-built noncollinear optical parametric amplifier. Another beam was passed through second harmonic generation beta barium borate crystal to generate the $400 \mathrm{~nm}$ pump pulse. The delay between the pump and probe was obtained by the motorized translation stage. The pump and probe beams are overlapped on to the sample from the same side. The transmitted probe beam was collected with an InGaAs dual-line array detector (Hamamatsu G11608-512). The transmission difference was read out by a custom-built lock-in board from Stresing Entwicklungsbüro.

\section{The time-of-flight secondary-ion mass spectroscopy (ToF-SIMS)}

ToF-SIMS analyses were carried out on an ION-TOF GmbH (Münster, Germany) TOF.SIMS 5 system. The instrument is equipped with a reflection type analyzer and microchannel plate detector. A Bi liquid metal ion source (LMIS) was employed for mass data acquisition. Mass data was acquired using the $\mathrm{Bi}^{3+}$ cluster ion. Mass data acquisition was performed by raster scanning over a $50 \times 50 \mu \mathrm{m}^{2}$ area. A $25 \mathrm{keV} \mathrm{Bi}^{3+}$ primary ion beam 40 delivering $0.18 \mathrm{pA}$ of current was used. A cycle time of $130 \mu \mathrm{s}$ was employed for mass data acquisition (with masses ranging from 1 to $1498 \mathrm{u}$ ). A Cs ion source was employed as the sputter/etch tool. The area analyzed by the LMIS was at the center of the sputter/etch crater formed using the rastered $\mathrm{Cs}^{+}$beam. A $3 \mathrm{kV} \mathrm{Cs}^{+}$primary ion beam was employed. The $\mathrm{Cs}^{+}$ sputter/etch area was $400 \times 400 \mu^{2}$. The depth profiling analyses were performed in the 'interlaced' mode, where the sources can operate in a simultaneous and continuous fashion. 


\section{Supplementary Text}

Identify the chemical composition of bright crystals

Elemental mapping via energy dispersive X-ray (EDX) analysis (fig. S1) shows that the bright crystals are attributed to lead halide complex (position 1), since the nitrogen and carbon elements in such a position are negligible or below the instrument detection limit. We also note that atomic ratio of $\left(\mathrm{I}^{-}+\mathrm{Br}^{-}\right) / \mathrm{Pb}^{2+}$ is approximated to 2 , in agreement with the stoichiometric ratios of $\mathrm{PbI}_{2}$ and $\mathrm{PbBr}_{2}$. Thus, we can conclude that the bright crystals present on the surface of the perovskite are $\mathrm{PbI}_{1.50} \mathrm{Br}_{0.50}$. On the contrary, the carbon and nitrogen were observed in the dark area (position 2), and the ratio of $\left(\mathrm{I}^{-}+\mathrm{Br}^{-}\right) / \mathrm{Pb}^{2+}$ was 2.66. Theoretically, the ratio of halide/lead in the metal-halide perovskite should be 3 . Herein, the ratio of lead/halide is between 2 and 3 , which is consistent with the excess $\mathrm{PbI}_{2}$ in the precursor solution.

\section{Urbach energy determined by PDS method}

We present Tauc plots derived from ultraviolet-visible (UV-Vis) absorption spectra in fig. S9A, showing an identical bandgap for both control and SSG films. Thus, we may expect improved device performance in the inverted planar heterojunction PSCs via enhancing the film quality. We also performed absorption measurements on perovskite samples coated on quartz substrates, using photothermal deflection spectroscopy (PDS) technique (fig. S9B), which gives 4-5 orders of magnitude dynamic range of sensitivity (39). The steepness of the below bandgap 20 absorption onset gives information about the electronic disorder in a semiconductor, with a steeper slope indicating lower disorder (40). This is typically quantified as the Urbach energy $\left(E_{\mathrm{u}}\right), A(E) \propto e^{E / E_{u}}$, where $A$ is the absorbance and $E$ is the excitation energy in electron volts. We present results in fig. S9B. We observe the average Urbach energy decrease from 16.0 to $14.2 \mathrm{meV}$ with the SSG, which is consistent with a reduction in the density of band tail states near the band edge that is responsible for electronic disorder.

\section{Modified Williamson-Hall method for microstrain estimation}

Broadening and shifts in the XRD peak can be caused by either a reduction in the grain size (Scherrer broadening) and/or non-uniform strain (microstrain). We note that Scherrer broadening 30 will only be significant when the grains are in the range of or less than $100 \mathrm{~nm}$, and as we will discuss later we do not expect this to be a significant contribution here. Strain is the relative change in size of an object with respect to its ideal size (or size before experiencing an external force). The microstrain in a crystalline material is a result of small fluctuations in the lattice spacing, induced by crystal imperfections/structural defects including dislocations, vacancies, stacking faults, interstitials, twinning and grain boundaries (41-43). By simply considering Braggs law for scattering of light of wavelength $\lambda, \mathrm{n} \lambda=2 d \sin \theta$, it is clear that small fluctuations in $d$ will result in small fluctuations, or broadening, in $\theta$ when measuring the X-ray diffraction from the material. We quantify the extent of microstrain in our perovskite films by analysing the peak broadening in the diffraction patterns according to the modified Williamson-Hall method $(42,44)$. The effective observed $d$-space broadening $\left(\Delta d_{\text {obs }}\right)$ determined by the XRD peak width broadening, is a convoluted function of the Gaussian full width half maximum broadening in the $2 \theta$ scan due to the instrument response ( $\left.\Delta d_{\text {ins }}\right)$, the grain size $\left(\Delta d_{\text {size }}\right)$ and the microstrain $\left(\Delta d_{\varepsilon}\right)$. These can be de-convoluted from the observed broadening, via, $\Delta d^{2}{ }_{\text {obs }}=\Delta d^{2}{ }_{\varepsilon}+\Delta d^{2}{ }_{\text {ins }}+\Delta d^{2}{ }_{\text {size, }}$, where the unit-less microstrain $\varepsilon$ is defined as $\varepsilon=\left(d_{\varepsilon} / d\right)$, where $\mathrm{d}$ is the mean $d$-spacing. For single crystals, the size effect induced peak width broadening can be neglected, hence if $d_{\text {size }}^{2}<<$ $d^{2}{ }_{\text {obs }}$ and we can write, $\left(\Delta d^{2}{ }_{\text {obs }}-\Delta d^{2}{ }_{\text {ins }}\right)^{1 / 2} \approx \varepsilon d$. Therefore, the slope of $\left(\Delta d^{2}{ }_{o b s}-\Delta d^{2}{ }_{\text {ins }}\right)^{1 / 2}$ versus $d$, 
gives the magnitude of the microstrain, $\varepsilon$, in the crystals (fig. S10A). In contrast to the pristine perovskite film, we can clearly observe a reduction in microstrain in the films with SSG process (fig. S10B). The SSG-G film shows the lowest microstrain value of $1.37 \%$. These small fluctuations in the lattice spacing are induced by the presence of crystal imperfections/structural defects including dislocations, vacancies, and stacking faults. It is plausible that the introduction of the SSG approach within the pristine films reduces the crystal defects at grain boundaries and on the top surface of the perovskite films, which can be highly favourable for the solar cell operation because the charge-trapping that happens at these locations may decrease.

Estimation of $V_{\text {oc }}$ limitations with different electronic disorders determined by PDS.

According to Beer-Lambert's approximation (assuming no reflection),

$$
I_{A}=I_{0}\left(1-e^{-\alpha t}\right)
$$

Where $I_{\mathrm{A}}$ is the current generated from the absorbed photons, $I_{0}$ is the current generated from incident photons, $\alpha$ is absorption coefficient and $t$ is photoactive layer thickness. We can therefore estimate external quantum efficiency (EQE) from absorption:

$$
\mathrm{EQE}=\frac{I_{A}}{I_{0}}=1-e^{-\alpha t}
$$

From the perspective of the reduced electronic disorder, which we inferred from the Urbach energy determining from the absorption spectra in fig. $\mathrm{S} 9 \mathrm{~B}$, we would expect an increase in $V_{\text {oc }}$. It is straight forward to quantify this expected increase in $V_{\mathrm{oc}}$ by converting the absorption spectrum into an assumed EQE spectrum, which we show in fig. S11.

For simplicity, current can be conveyed as: $\mathrm{I}=$ \#photon absorbed - \# photon emitted Therefore,

$$
I=\int E Q E(\lambda) \cdot \operatorname{AM} 1.5(\lambda) \cdot d \lambda-\int e^{\frac{q V}{k T}}
$$

Where, $e^{\frac{q V}{k T}}$ is obtained from the Planck's Law.

We calculated the $V_{\mathrm{OC}}$ in the radiative limit with the optoelectronic reciprocity relation theory $(45,46)$. The short-circuit photocurrent $\left(J_{\mathrm{Sc}}\right)$ is derived by integrating the photovoltaic external quantum efficiency (EQEPV) multiplied with the photon flux of the solar spectrum $\left(\Phi_{\mathrm{AM} 1.5}\right)$ over all photon energies as:

$$
J_{S C}=q \int E Q E_{P V}(E) \cdot \Phi_{A M 1.5}(E) \cdot d E
$$

Furthermore, in the dark, all absorbed photons from the environment (assumed photon flux from a black body at $300 \mathrm{~K} \Phi_{\mathrm{BB}}$ ) are reemitted due to energy conservation. Following Shockley's diode law, under a given bias voltage the probability of the reemission can be described by FermiDirac statistics and approximated with a Boltzmann term. The dark saturation current can then be described by the electroluminescence external quantum efficiency $(E R E)$ and the $E Q E_{\mathrm{PV}}$ of the device:

$$
J_{0}(V)=\frac{q}{E R E} \int E Q E_{P V}(E) \cdot \Phi_{B B}(E) \cdot d E\left(e^{\frac{q V}{K_{B} T}}-1\right)
$$


Combining both currents leads to the current-voltage relationship $J(V)=J_{\mathrm{sc}}-J_{0}(V)$. If we set $J=0$ and solve the equation for voltage, we end up with the $V_{\mathrm{oc}}$ :

$$
V_{o c}=\frac{K_{B} T}{q} \ln \left(\frac{J_{s c}}{J_{0}}+1\right)
$$

In the radiative limit, we assume zero nonradiative recombination and can therefore set ERE $=1$, to get the $V_{\mathrm{oc}}$ in the radiative limit:

$$
V_{o c, \text { rad }}=\frac{K_{B} T}{q} \ln \left(\frac{\int E Q E_{P V}(E) \cdot \Phi_{A M 1.5}(E) \cdot d E}{\int E Q E_{P V}(E) \cdot \Phi_{B B}(E) \cdot d E}+1\right)
$$

We therefore calculate the $V_{\mathrm{oc}}$ limit for the control and SSG-G processed devices to be $1.30 \mathrm{~V}$ and $1.31 \mathrm{~V}$, respectively.

Estimation of $V_{\text {oc }}$ from external radiative efficiency (ERE).

Using the EL spectrum, the EQE and the reciprocity relation (14), we are able to determine the theoretical radiative limit of the $V_{\mathrm{oc}}\left(V_{\mathrm{oc}}, \mathrm{rad}\right)$ to be $1.33 \sim 1.34 \mathrm{~V}$. However, in a real solar cell, additional nonradiative losses need be considered, thus the $\Delta V_{\mathrm{oc}}$, rad can then be calculated with equation.

$\Delta V_{o c, r a d}=\left(k_{B} T\right) / q \ln [E R E]$

Where the ERE is the electroluminescent external quantum efficiency of the solar cell, $k_{\mathrm{B}}$ is a Boltzmann constant and $T$ is a temperature. As the temperature is $300 \mathrm{~K}$, the $V_{\text {oc }}$ can be predicted with the following equation by yielding the ERE value (5).

$V_{o c}=V_{o c, r a d}-\Delta V_{o c, r a d}=V_{o c, r a d}+60 \mathrm{mV} \cdot \log E R E_{, T=300 \mathrm{~K}}$

We have measured the ERE of an SSG-G solar cell at the point where the injection current 20 is equal to photo-generation current under short-circuit, and we measure the ERE of 1.18\%. This leads to $\Delta V_{\mathrm{oc}}$, rad $=115 \mathrm{mV}$ and thus we estimate a calculated $V_{\mathrm{oc}}$ of $1.21 \mathrm{~V}$ that matches with the results from the $J-V$ scans of the solar cell. 


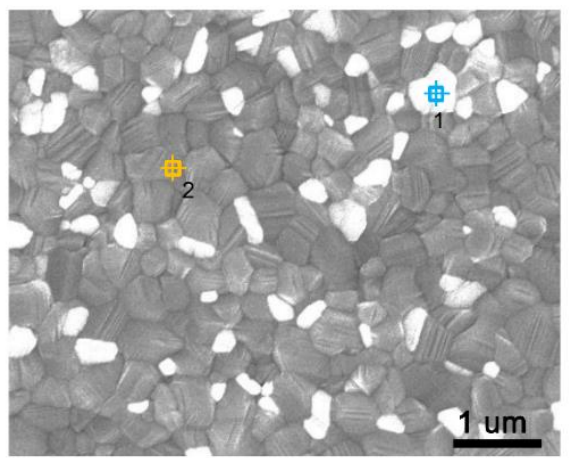

\begin{tabular}{|l|l|l|l|l|l|}
\hline & C-K & N-K & Br-L & I-L & Pb-M \\
\hline 1 & 0.00 & & 13.00 & 38.57 & 22.71 \\
\hline 2 & 16.81 & 10.11 & 6.38 & 29.47 & 13.47 \\
\hline
\end{tabular}

Fig. S1. SEM image and EDX analysis. Identify the chemical composition of the bright crystals. 


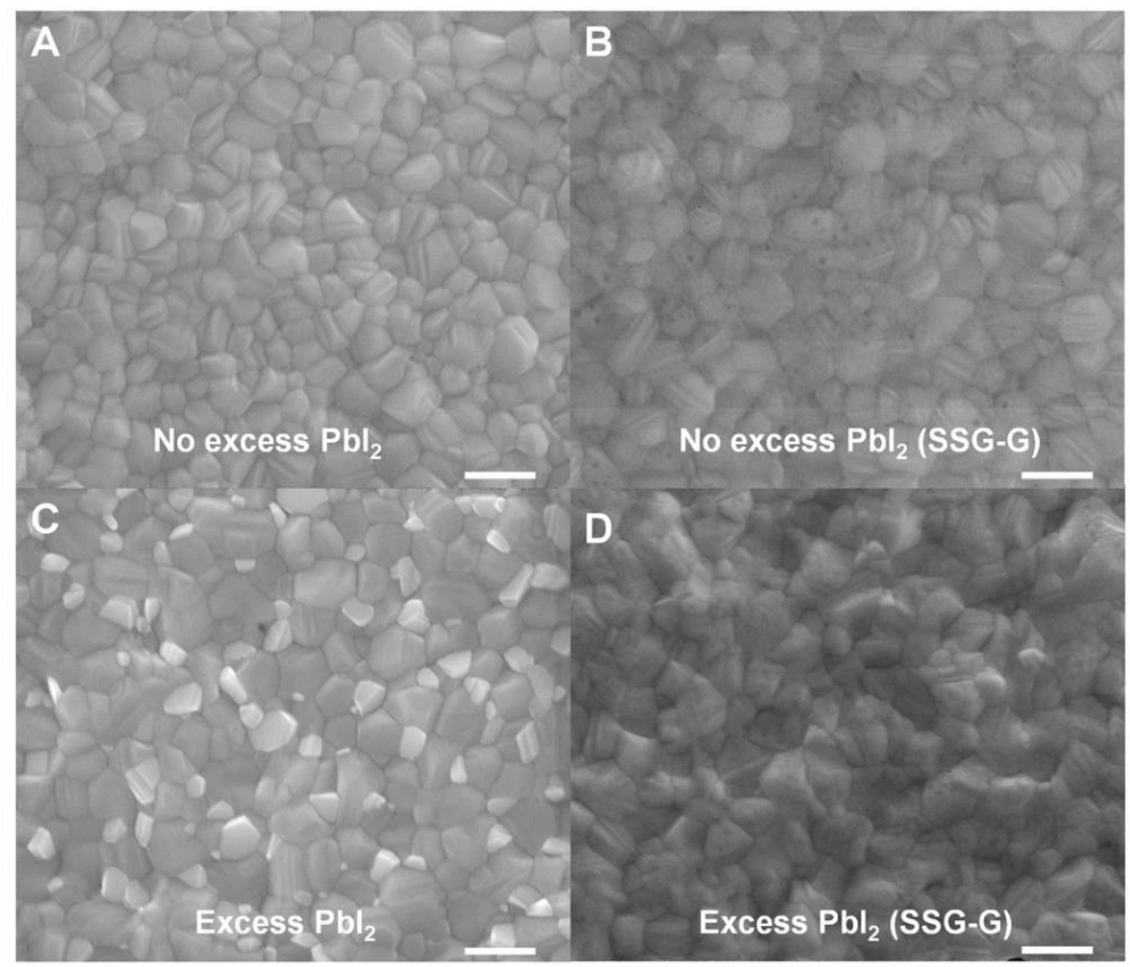

Fig. S2. Top-view SEM images of surface morphologies for the films with and without the excess $\mathbf{P b I}_{2}$. The films processed from the solution containing no excess $\mathrm{PbI}_{2}$ : without (A) and with (B) the SSG-process. The films processed from the solution containing the excess $\mathrm{PbI}_{2}$ $(5 \%)$ : without $(\mathbf{C})$ and with (D) the SSG-G process. The scale bar is $500 \mathrm{~nm}$. The excess bright crystals presenting on the surface (grain boundaries) have led to a secondary growth based on $\mathrm{GABr}$ organic salts. 


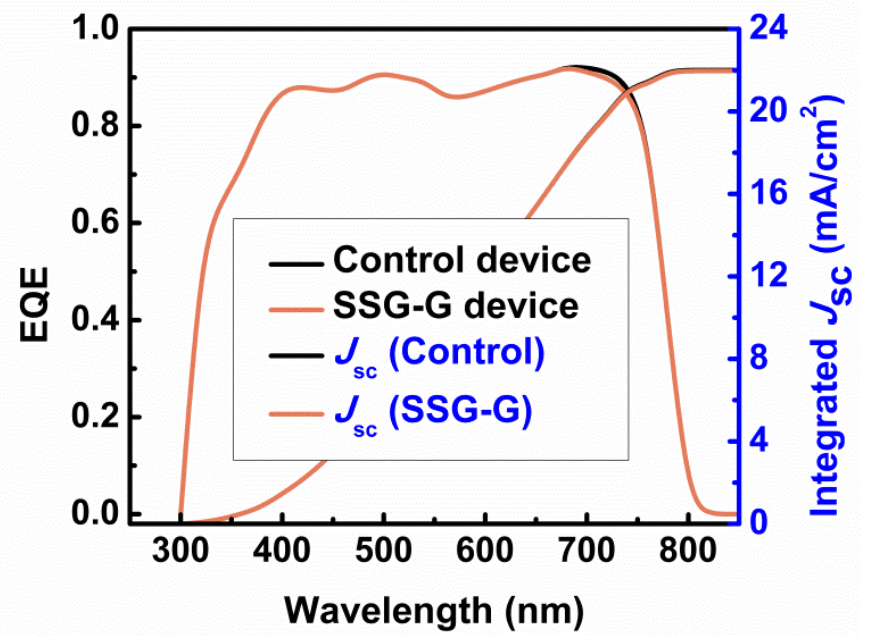

Fig. S3. EQE spectra of cells based on solid polycrystalline perovskite films without (control) and with the SSG-G process. The EQE spectra for both devices exhibiting the similar characteristics, and integrated $J_{\mathrm{sc}}$ values obtained from the EQE were around $22 \mathrm{~mA} \mathrm{~cm}{ }^{-2}(22.0$ $\mathrm{mA} \mathrm{cm}^{-2}$ for the control device and $21.96 \mathrm{~mA} \mathrm{~cm}^{-2}$ for the SSG-G device). 


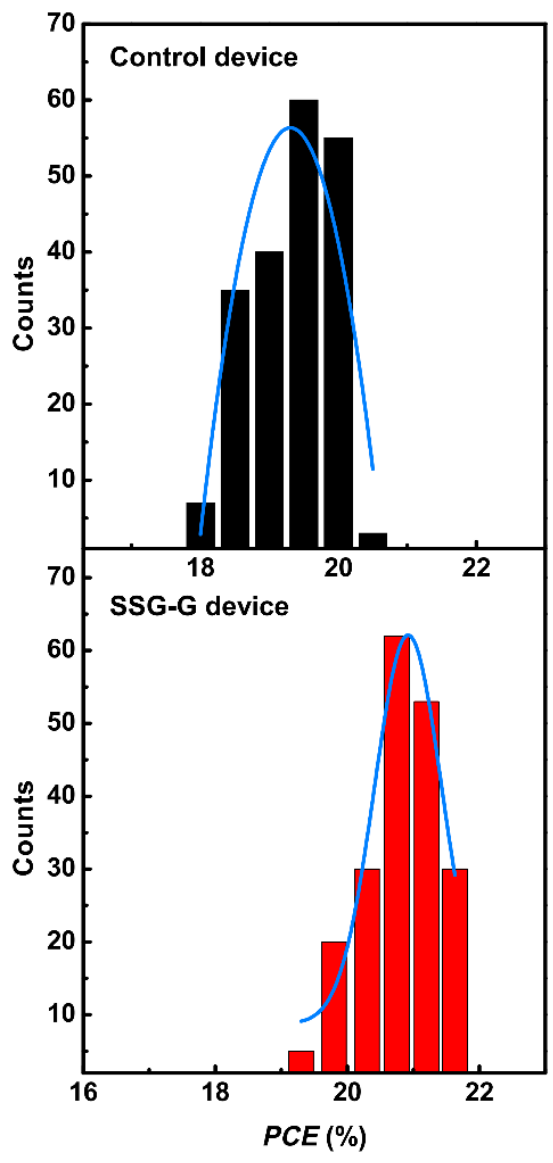

Fig. S4. Histograms of the PCEs for 200 devices prepared without (control) and with the SSG-G method. The Gaussian fits have been added to aid the eye. 


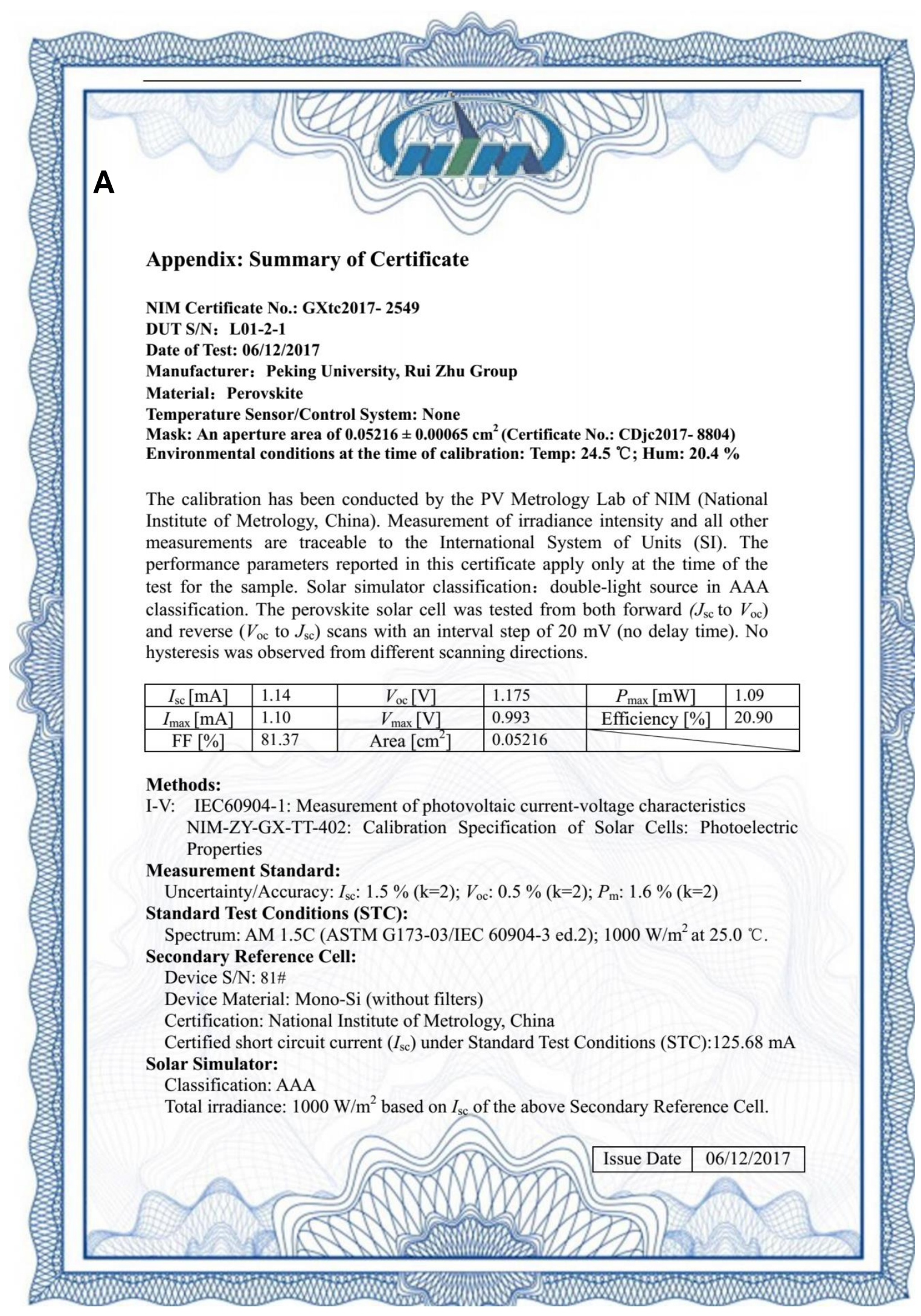




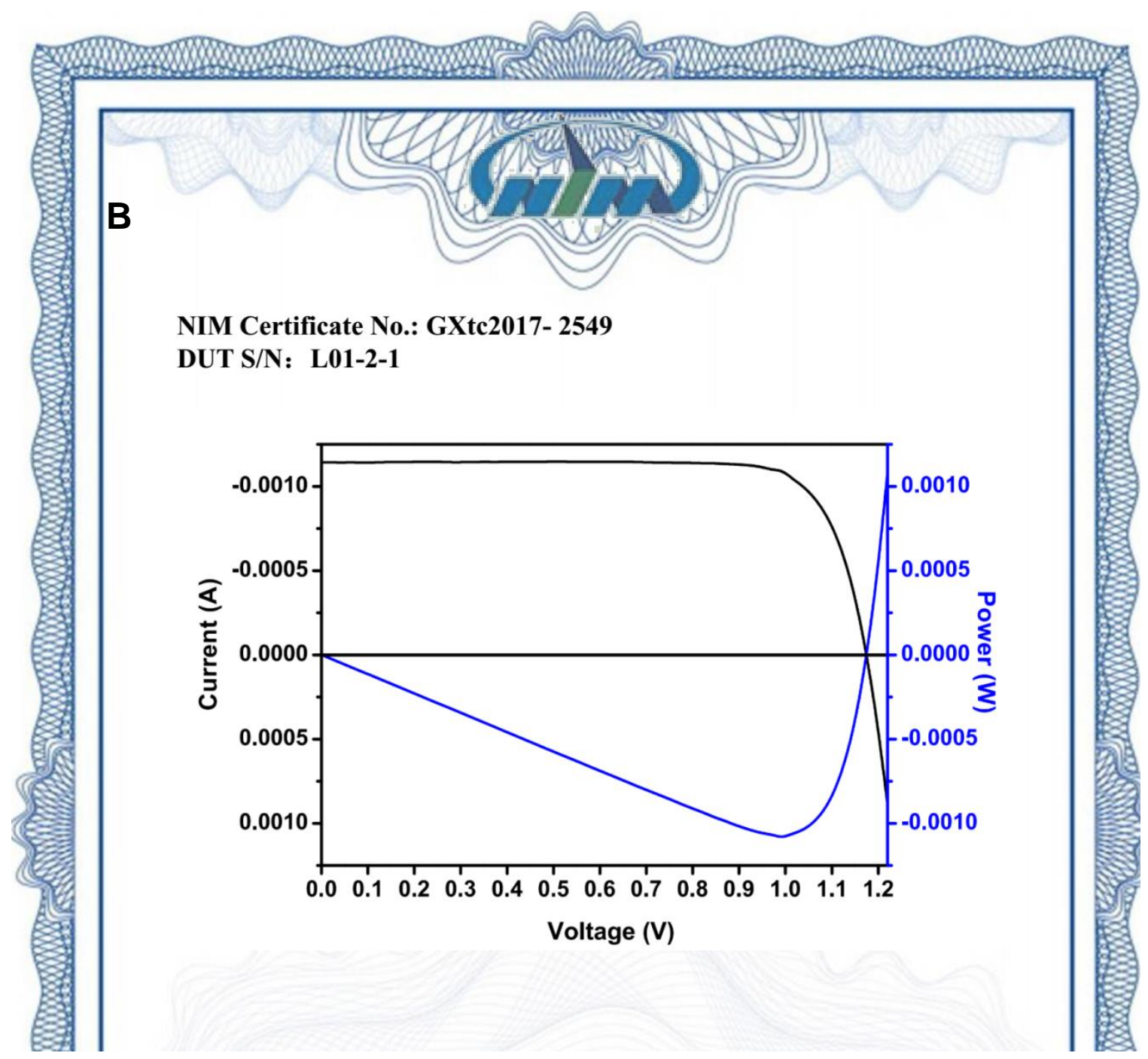

Fig. S5. An independent testing report. (A and B) Independent PCE testing report of an inverted planar heterojunction PSC by an accredited PV Metrology Laboratory of NIM (National Institute of Metrology, China) verified a PCE of $20.90 \%$ (a $J_{\text {sc }}$ of $21.86 \mathrm{~mA} \mathrm{~cm}^{-2}$, a $V_{\text {oc }}$ of 1.175 $\mathrm{V}$, and a FF of $81.37 \%$ ) with negligible hysteresis. The cell was tested in air without encapsulation or protection during the test process. 


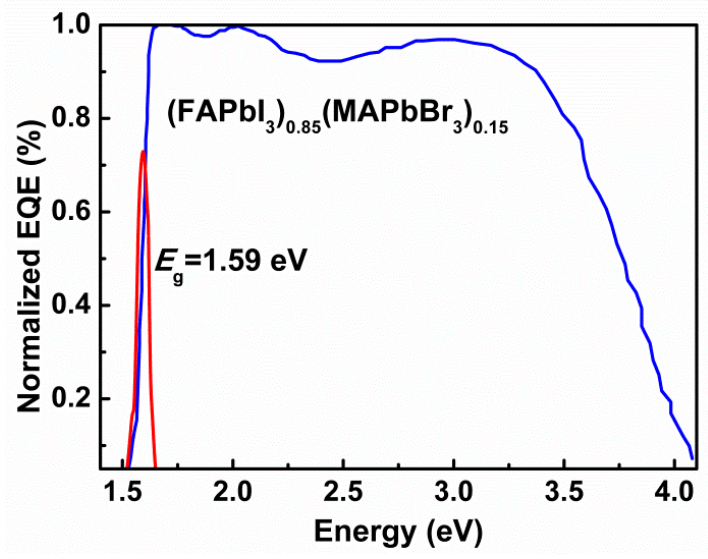

Fig. S6. The normalized EQE. The EQE spectrum extracted from the data reported by Zheng et al. [Adapted from (3) with permission]. We have determined the bandgap from this EQE plot to be $1.59 \mathrm{eV}$, via the differential of the EQE spectrum. 

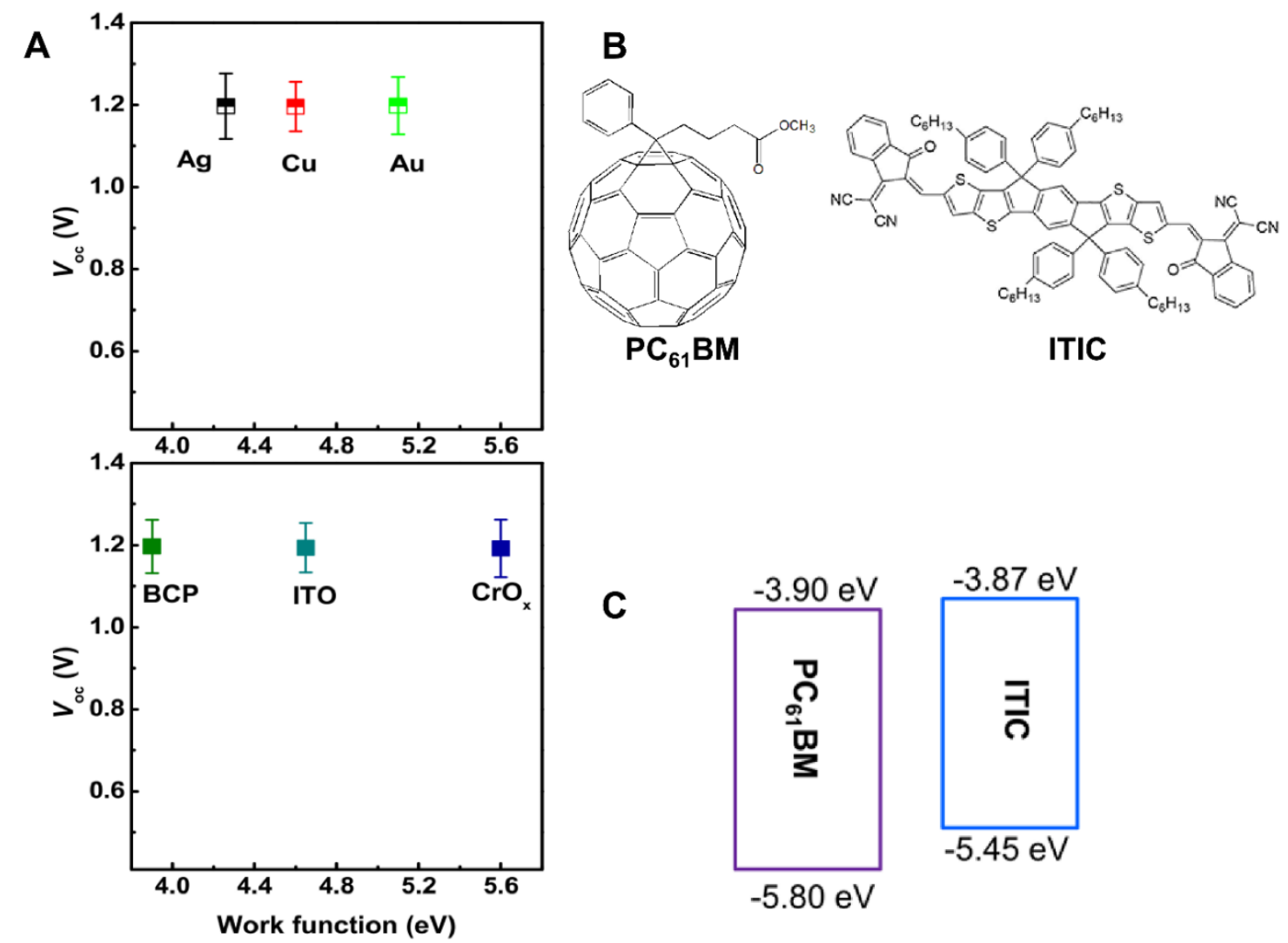

Fig. S7. Factors affecting the $V_{\text {oc }}$ (A) The $V_{\text {oc }}$ dependence of the work functions of metal electrodes and buffer layers for the inverted planar heterojunction PSCs processed from the SSG$\mathrm{G}$ process. (B) The molecular structures of $\mathrm{PC}_{61} \mathrm{BM}$ and ITIC electron-extraction layers. (C) Energy level diagrams for the $\mathrm{PC}_{61} \mathrm{BM}$ and ITIC. Summary of device performances is shown in table S8. 


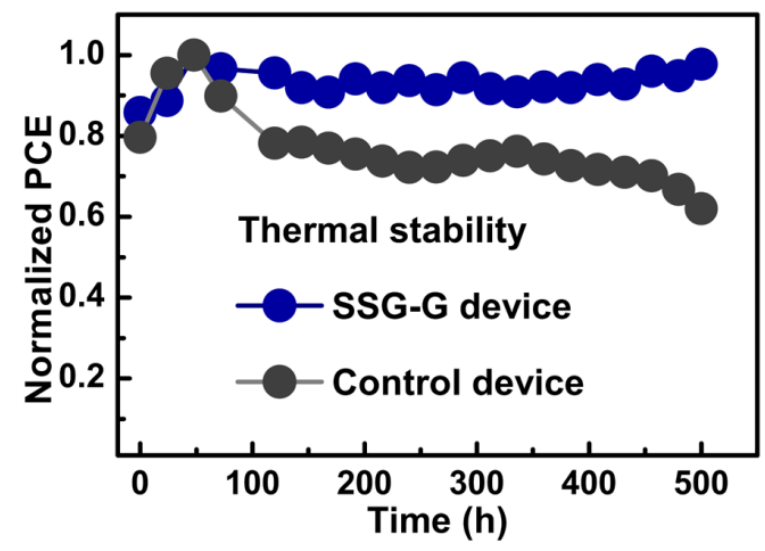

Fig. S8. Thermal stability. Thermal-stability test was performed for the non-encapsulated cells (typical control and SSG-G devices) aging for $500 \mathrm{~h}$ at $85^{\circ} \mathrm{C}$ in a nitrogen atmosphere. 

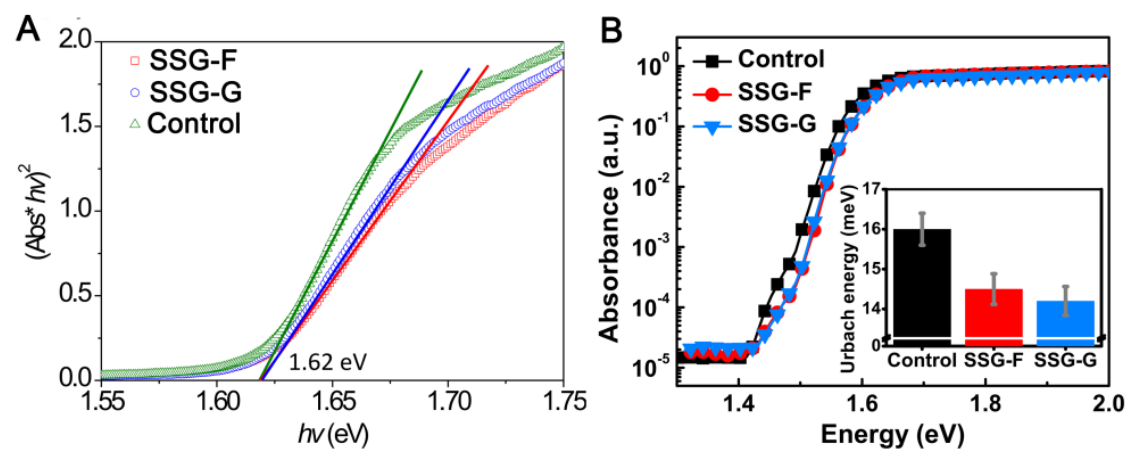

Fig. S9. Tauc plots and PDS spectra. (A) Tauc plots of the solid perovskite thin films prepared without (control) and with the SSG method. (B) PDS spectra for thin films with and without the SSG process. Inset is Urbach energy extracted from the PDS measurements. 

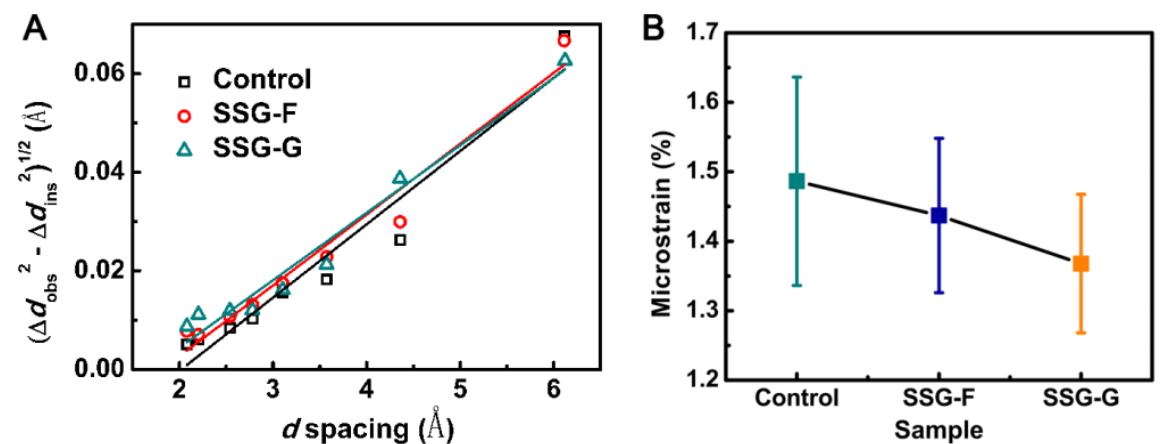

Fig. S10. Estimated microstrain. (A) A modified Williamson-Hall plot of $\left(\Delta d_{\mathrm{obs}}{ }^{2}-\Delta d_{\mathrm{ins}}{ }^{2}\right)^{1 / 2}$ versus $d$ spacing extracted from the corresponding diffraction profiles. The slopes of the solid lines from linear fits indicate the extents of microstrain for the different films. (B) Calculated microstrain from the XRD patterns. 


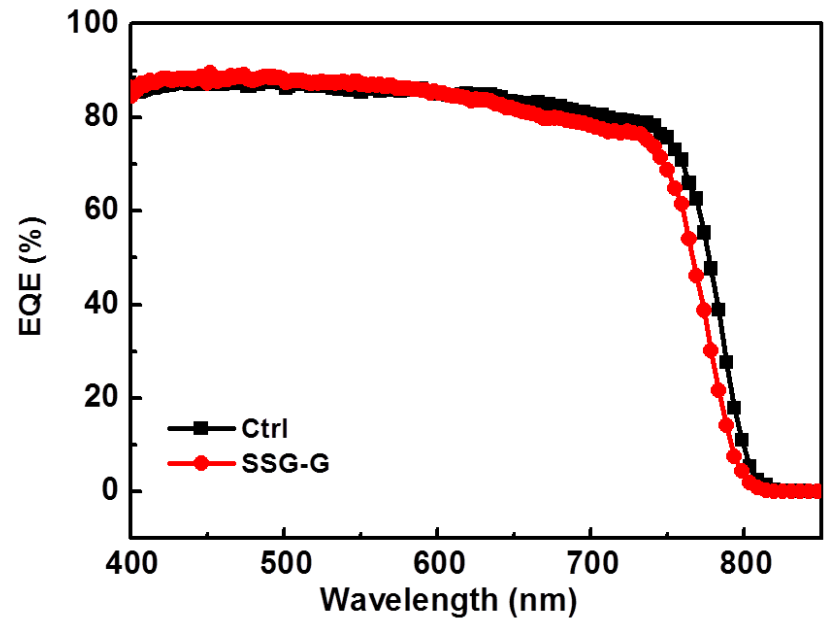

Fig. S11. Calculated EQE spectra using the absorbance spectra in fig. S9B. 


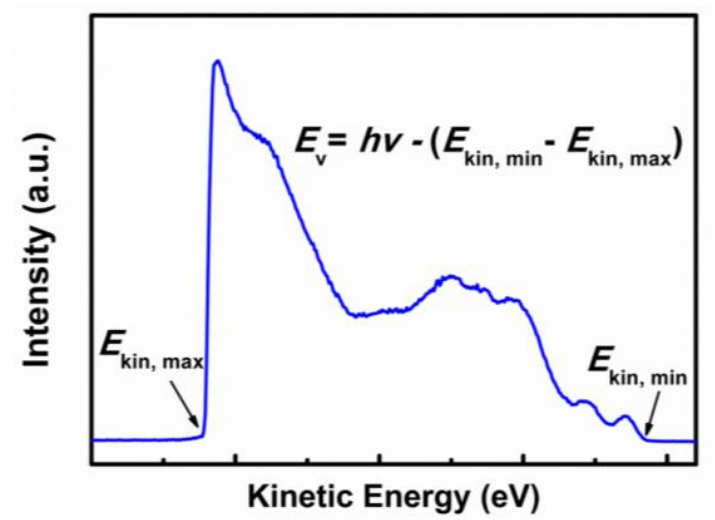

Fig. S12. Valence band spectrum. Valence band spectrum of the solid polycrystalline perovskite films coated on the ITO substrates, and corresponding $E_{\mathrm{kin}, \max }$ and $E_{\mathrm{kin}, \min }$ positions are indicated. The valence-band edge $\left(E_{\mathrm{v}}\right)$ can be obtained from the equation as follows: $E_{\mathrm{v}}=h v-$ $\left(E_{\text {kin, } \min }-E_{\text {kin, max }}\right)$. 

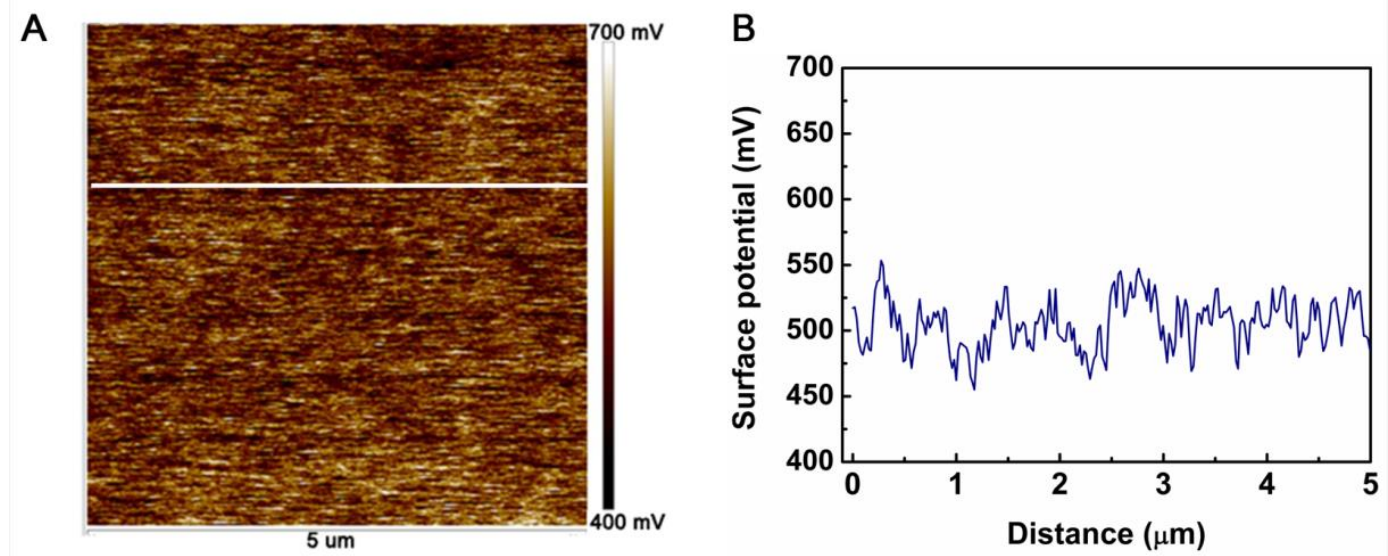

Fig. S13. Surface potential image. (A) The 2D mapping of Kelvin probe force microscopy for the SSG-G film. (B) The variation of surface potential along the white line marked in 2D mapping. 

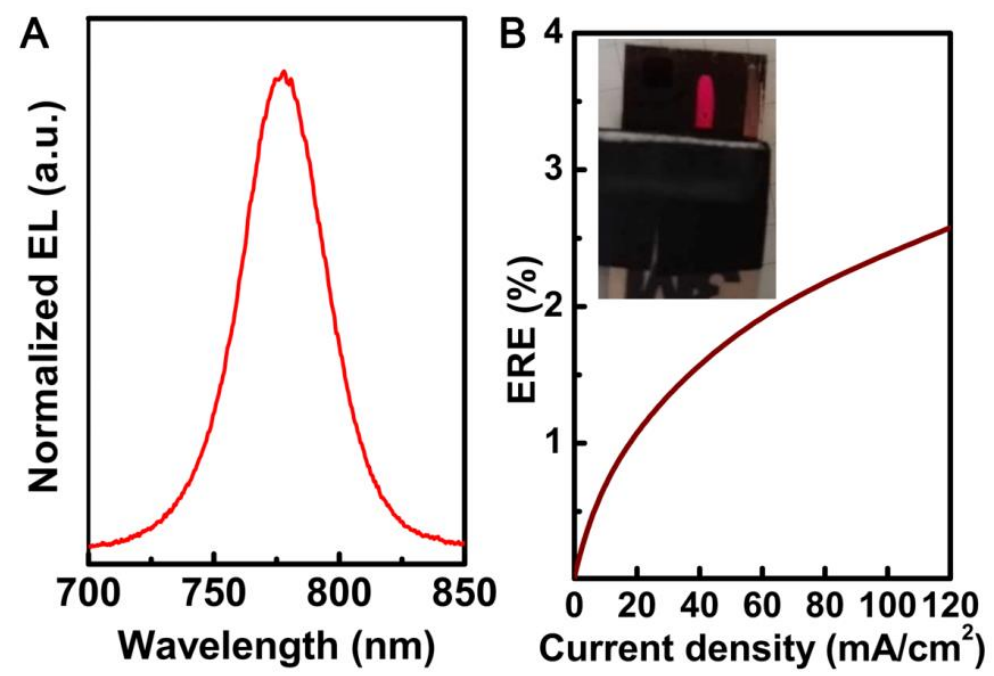

Fig. S14. Electroluminescence (EL) and external radiative efficiency (ERE): The normalized EL (A) and ERE spectra (B) of a solar cell as a LED. The inset clearly shows a bright emission by applying a forward voltage of $2 \mathrm{~V}$. 

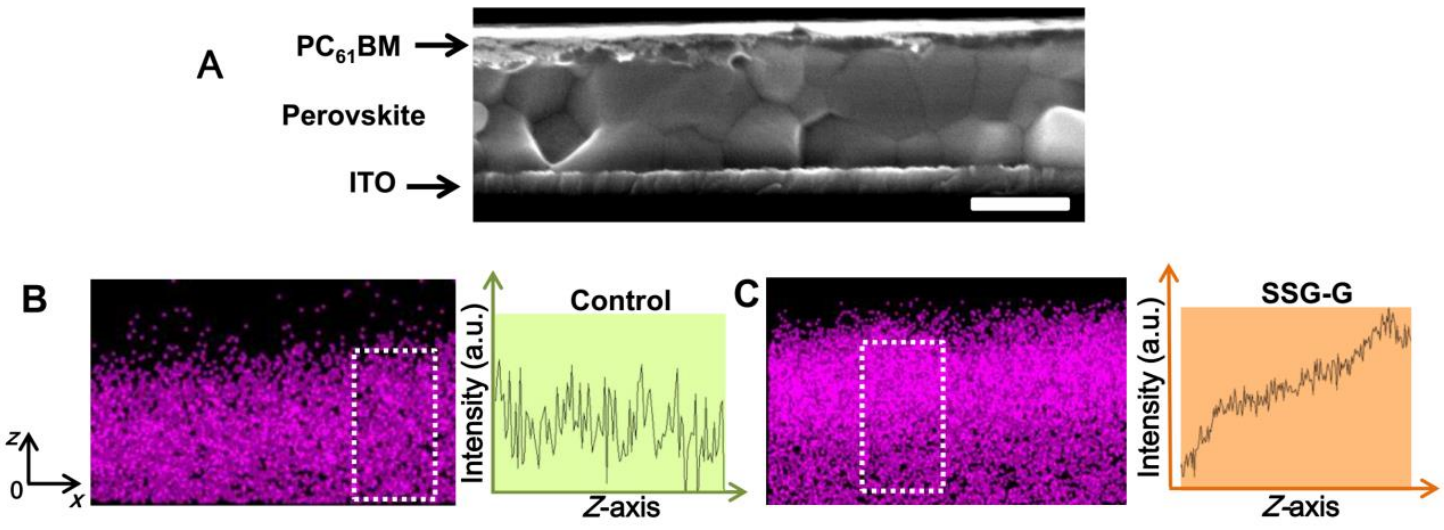

Fig. S15. Cross-sectional SEM image and EDX analysis: (A) Cross-sectional SEM image of the perovskite thin film obtained with the SSG-G process. The scale bar is $500 \mathrm{~nm}$. (B and C) EDX analysis (bromide) of the cross-sectional area of the perovskite films on ITO substrates. The dot-square areas $(265 \mathrm{~nm} \times 530 \mathrm{~nm})$ were used to extract mean bromide-distribution profile along $Z$-axis direction (from bottom side to perovskite surface): (B) control and (C) SSG-G film. 

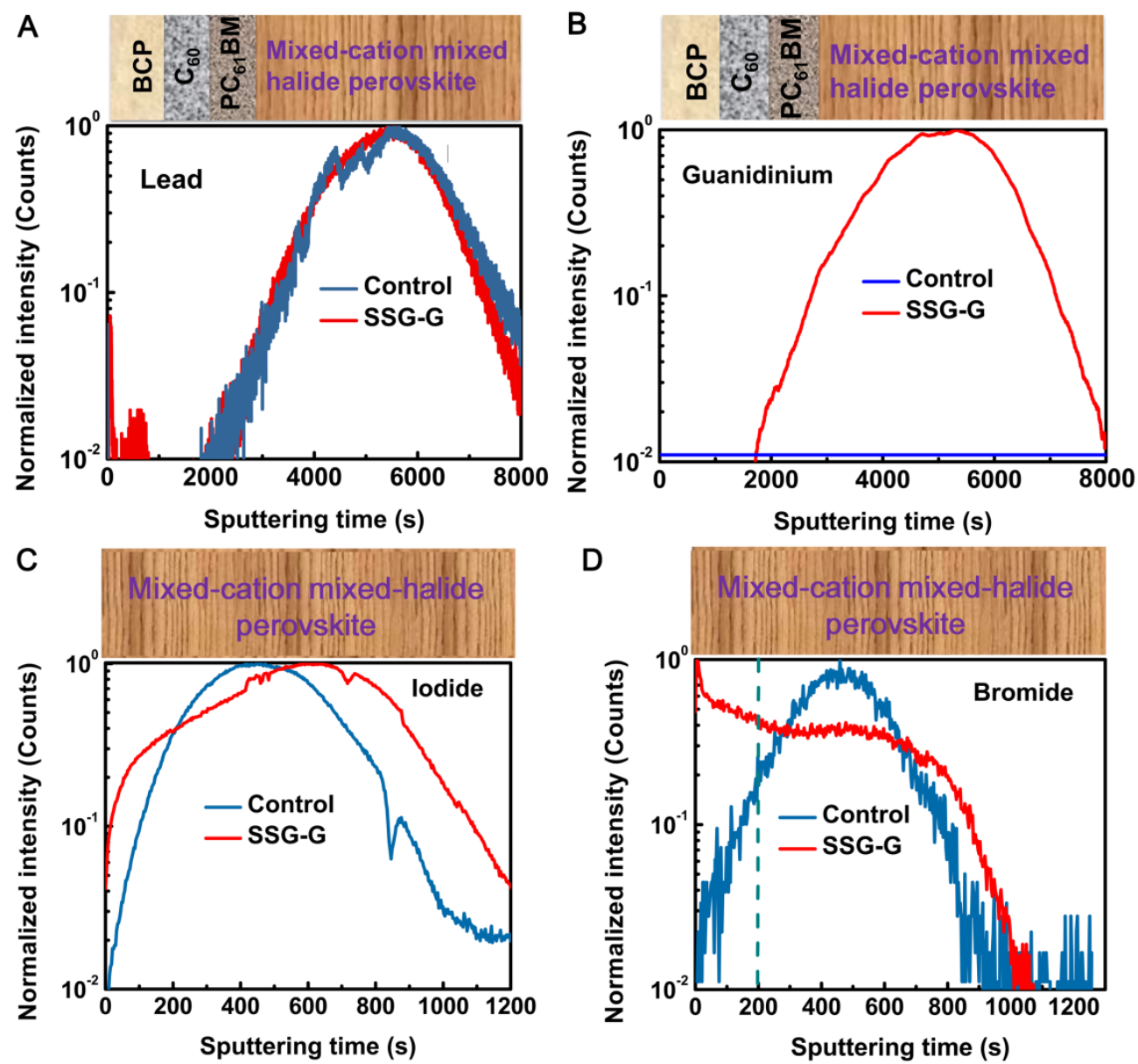

Fig. S16. Elemental depth profile: the films were obtained without (control) and with SSG-G process, respectively. Cations distribution across the varied layers: $(\mathbf{A})$ lead cation $\left(\mathrm{Pb}^{2+}\right)$ and $(\mathbf{B})$ organic cation $\left(\mathrm{GA}^{+}\right)$. Halide depth profiles across the perovskite layer: $(\mathbf{C})$ iodide $\left(\mathrm{I}^{-}\right)$and $(\mathbf{D})$ bromide $\left(\mathrm{Br}^{-}\right)$. Note that the difference of sputtering time is due to the different film structures and measurement rates for the cation and anion. 
Table S1.

Comparisons of device performances for the inverted planar heterojunction PSCs with and without excess $\mathrm{PbI}_{2}$, and corresponding devices prepared with and without the SSG process. The

5 devices were measured from reverse scans (RS, from $1.22 \mathrm{~V}$ to $-0.02 \mathrm{~V}$ ) under simulated AM $1.5 \mathrm{G}$ solar irradiation at $100 \mathrm{~mW} \mathrm{~cm}^{-2}$.

\begin{tabular}{|c|c|c|c|c|c|}
\hline $\begin{array}{c}\text { Excess } \\
\mathrm{PbI}_{2}\end{array}$ & SSG & $\begin{array}{c}V_{\mathrm{oc}} \\
(\mathrm{V})\end{array}$ & $\begin{array}{c}J_{\mathrm{sc}} \\
\left(\mathrm{mA} \mathrm{cm}^{-2}\right)\end{array}$ & FF & $\begin{array}{c}\text { PCE } \\
(\%)\end{array}$ \\
\hline $\mathrm{W} / \mathrm{O}$ & $\mathrm{W} / \mathrm{O}$ & $1.08 \pm 0.02$ & $22.71 \pm 1.15$ & $0.78 \pm 0.02$ & $19.13 \pm 0.86$ \\
\hline $\mathrm{W} / \mathrm{O}$ & $\mathrm{W}$ & $1.13 \pm 0.02$ & $22.67 \pm 0.96$ & $0.78 \pm 0.03$ & $19.98 \pm 0.47$ \\
\hline $\mathrm{W}$ & $\mathrm{W} / \mathrm{O}$ & $1.10 \pm 0.03$ & $22.70 \pm 1.06$ & $0.78 \pm 0.02$ & $19.48 \pm 0.87$ \\
\hline $\mathrm{W}$ & $\mathrm{W}$ & $1.18 \pm 0.02$ & $22.55 \pm 0.84$ & $0.78 \pm 0.02$ & $20.76 \pm 0.45$ \\
\hline
\end{tabular}




\section{Table S2.}

Solar cell performance parameters obtained from the devices based on SSG-F (FABr) process under simulated AM 1.5G solar irradiation at $100 \mathrm{~mW} \mathrm{~cm}$. The devices were measured from reverse scans (RS, from $1.22 \mathrm{~V}$ to $-0.02 \mathrm{~V}$ ).

\begin{tabular}{|c|c|c|c|c|c|}
\hline $\begin{array}{c}\text { SSG-F } \\
\left(\mathrm{mg} \mathrm{ml}^{-1}\right)\end{array}$ & $\begin{array}{c}\text { Scan } \\
\text { direction }\end{array}$ & $\begin{array}{c}V_{\text {oc }} \\
(\mathrm{V})\end{array}$ & $\begin{array}{c}J_{s c} \\
\left(\mathrm{~mA} \mathrm{~cm}^{-2}\right)\end{array}$ & FF & $\begin{array}{c}\text { PCE } \\
(\%)\end{array}$ \\
\hline 0 & $\mathrm{RS}$ & $1.10 \pm 0.03$ & $22.65 \pm 1.03$ & $0.79 \pm 0.01$ & $19.68 \pm 0.87$ \\
\hline 4.0 & $\mathrm{RS}$ & $1.17 \pm 0.02$ & $22.65 \pm 0.99$ & $0.75 \pm 0.02$ & $19.88 \pm 0.60$ \\
\hline 4.5 & $\mathrm{RS}$ & $1.18 \pm 0.02$ & $22.67 \pm 0.97$ & $0.76 \pm 0.02$ & $20.33 \pm 0.47$ \\
\hline 5.0 & $\mathrm{RS}$ & $1.19 \pm 0.02$ & $22.54 \pm 1.00$ & $0.78 \pm 0.02$ & $20.92 \pm 0.47$ \\
\hline 5.5 & $\mathrm{RS}$ & $1.20 \pm 0.02$ & $22.35 \pm 0.99$ & $0.77 \pm 0.03$ & $20.65 \pm 0.46$ \\
\hline 6.0 & $\mathrm{RS}$ & $1.20 \pm 0.02$ & $22.15 \pm 0.97$ & $0.71 \pm 0.02$ & $18.87 \pm 0.45$ \\
\hline
\end{tabular}




\section{Table S3.}

Solar cell performance parameters obtained from the devices based on SSG-G (GABr) process under simulated AM 1.5G solar irradiation at $100 \mathrm{~mW} \mathrm{~cm}$. The devices were measured from reverse scans (RS, from $1.22 \mathrm{~V}$ to $-0.02 \mathrm{~V}$ ).

\begin{tabular}{|c|c|c|c|c|c|}
\hline $\begin{array}{c}\text { SSG-G } \\
\left(\mathrm{mg} \mathrm{m}^{-1}\right)\end{array}$ & $\begin{array}{c}\text { Scan } \\
\text { direction }\end{array}$ & $\begin{array}{c}V_{\mathrm{oc}} \\
(\mathrm{V})\end{array}$ & $\begin{array}{c}J_{s c} \\
\left(\mathrm{~mA} \mathrm{~cm}^{-2}\right)\end{array}$ & FF & $\begin{array}{c}\text { PCE } \\
(\%)\end{array}$ \\
\hline 0 & $\mathrm{RS}$ & $1.10 \pm 0.03$ & $22.62 \pm 1.01$ & $0.79 \pm 0.01$ & $19.66 \pm 0.87$ \\
\hline 4.5 & $\mathrm{RS}$ & $1.18 \pm 0.01$ & $22.61 \pm 0.97$ & $0.75 \pm 0.02$ & $20.01 \pm 0.51$ \\
\hline 5.0 & $\mathrm{RS}$ & $1.20 \pm 0.02$ & $22.43 \pm 0.99$ & $0.78 \pm 0.07$ & $20.99 \pm 0.46$ \\
\hline 5.5 & $\mathrm{RS}$ & $1.20 \pm 0.02$ & $22.31 \pm 1.00$ & $0.77 \pm 0.02$ & $20.61 \pm 0.46$ \\
\hline 6.0 & $\mathrm{RS}$ & $1.20 \pm 0.02$ & $21.41 \pm 1.06$ & $0.74 \pm 0.03$ & $19.01 \pm 0.52$ \\
\hline
\end{tabular}




\section{Table S4.}

The optimized temperature of thermal annealing for the devices based on SSG-G process. The devices were measured from reverse scans (RS, from $1.22 \mathrm{~V}$ to $-0.02 \mathrm{~V}$ ) under simulated AM $1.5 \mathrm{G}$ solar irradiation at $100 \mathrm{~mW} \mathrm{~cm}^{-2}$.

\begin{tabular}{|c|c|c|c|c|c|}
\hline $\begin{array}{c}\text { Temperature } \\
\left({ }^{\circ} \mathrm{C}\right)\end{array}$ & $\begin{array}{c}\text { Scan } \\
\text { direction }\end{array}$ & $\begin{array}{c}V_{\mathrm{oc}} \\
(\mathrm{V})\end{array}$ & $\begin{array}{c}J_{s c} \\
\left(\mathrm{~mA} \mathrm{~cm}^{-2}\right)\end{array}$ & FF & $\begin{array}{c}\text { PCE } \\
(\%)\end{array}$ \\
\hline 95 & RS & $1.19 \pm 0.01$ & $22.72 \pm 0.99$ & $0.71 \pm 0.02$ & $19.20 \pm 0.59$ \\
\hline 100 & RS & $1.20 \pm 0.02$ & $22.52 \pm 0.99$ & $0.76 \pm 0.02$ & $20.54 \pm 0.52$ \\
\hline 105 & RS & $1.20 \pm 0.02$ & $22.48 \pm 1.00$ & $0.77 \pm 0.02$ & $20.77 \pm 0.45$ \\
\hline 110 & RS & $1.20 \pm 0.02$ & $22.51 \pm 1.02$ & $0.76 \pm 0.02$ & $20.53 \pm 0.46$ \\
\hline 115 & RS & $1.20 \pm 0.02$ & $22.45 \pm 1.06$ & $0.73 \pm 0.03$ & $19.67 \pm 0.47$ \\
\hline
\end{tabular}




\section{Table S5.}

Solar cell performance parameters obtained from the devices based on SSG process (FAI, FABr, $\mathrm{GAI}$ and $\mathrm{GABr}$ ). The devices were measured from reverse scans (RS, from $1.22 \mathrm{~V}$ to $-0.02 \mathrm{~V}$ ) under simulated AM 1.5G solar irradiation at $100 \mathrm{~mW} \mathrm{~cm}^{-2}$. Note that yellow colour films (nonperovskite phase) were obtained when the pristine perovskite films were sequentially grown with SSG process based $\mathrm{FACl}$ and $\mathrm{GACl}$.

\begin{tabular}{|c|c|c|c|c|c|}
\hline SSG & $\begin{array}{c}\text { Scan } \\
\text { direction }\end{array}$ & $\begin{array}{c}V_{\text {oc }} \\
(\mathrm{V})\end{array}$ & $\begin{array}{c}J_{s c} \\
\left(\mathrm{~mA} \mathrm{~cm}^{-2}\right)\end{array}$ & FF & $\begin{array}{c}\text { PCE } \\
(\%)\end{array}$ \\
\hline FAI & RS & $1.10 \pm 0.02$ & $22.73 \pm 0.91$ & $0.79 \pm 0.02$ & $19.75 \pm 0.53$ \\
\hline FABr & RS & $1.19 \pm 0.01$ & $22.65 \pm 0.65$ & $0.77 \pm 0.02$ & $20.75 \pm 0.48$ \\
\hline GAI & RS & $1.15 \pm 0.02$ & $22.69 \pm 0.92$ & $0.76 \pm 0.02$ & $19.83 \pm 0.69$ \\
\hline GABr & RS & $1.20 \pm 0.02$ & $22.50 \pm 0.05$ & $0.78 \pm 0.01$ & $21.06 \pm 0.45$ \\
\hline FACl or GACl & ----- & ----- & ----- & ----- & ----- \\
\hline
\end{tabular}




\section{Table S6.}

Solar cell performance parameters obtained from the devices based on solid perovskite films with and without the SSG-G process. The devices were measured from both reverse (from 1.22 $\mathrm{V}$ to $-0.02 \mathrm{~V}$, no delay time, RS) and forward scans (from $-0.02 \mathrm{~V}$ to $1.22 \mathrm{~V}$, no delay time, FS) under simulated AM $1.5 \mathrm{G}$ solar irradiation at $100 \mathrm{~mW} \mathrm{~cm}^{-2}$.

\begin{tabular}{|c|c|c|c|c|c|}
\hline Device & $\begin{array}{c}\text { Scan } \\
\text { direction }\end{array}$ & $\begin{array}{c}V_{\text {oc }} \\
(\mathrm{V})\end{array}$ & $\begin{array}{c}J_{s c} \\
\left(\mathrm{~mA} \mathrm{~cm}^{-2}\right)\end{array}$ & FF & $\begin{array}{c}\text { PCE } \\
(\%)\end{array}$ \\
\hline \multirow{3}{*}{ Control } & RS & $1.10 \pm 0.03$ & $22.53 \pm 0.98$ & $0.79 \pm 0.03$ & $19.58 \pm 0.86$ \\
\cline { 2 - 6 } & FS & $1.10 \pm 0.02$ & $22.59 \pm 0.96$ & $0.78 \pm 0.01$ & $19.38 \pm 0.85$ \\
\cline { 2 - 6 } & Average & $1.10 \pm 0.03$ & $22.56 \pm 0.97$ & $0.785 \pm 0.02$ & $19.48 \pm 0.85$ \\
\hline \multirow{3}{*}{ SSG-G } & RS & $1.20 \pm 0.01$ & $22.48 \pm 0.89$ & $0.77 \pm 0.01$ & $20.77 \pm 0.47$ \\
\cline { 2 - 7 } & FS & $1.20 \pm 0.02$ & $22.51 \pm 0.95$ & $0.77 \pm 0.01$ & $20.80 \pm 0.52$ \\
\cline { 2 - 6 } & Average & $1.20 \pm 0.02$ & $22.50 \pm 0.92$ & $0.77 \pm 0.01$ & $20.79 \pm 0.50$ \\
\hline
\end{tabular}




\section{Table S7.}

Summary of some top performances for inverted planar heterojunction PSCs based on similar band-gap perovskites in recent literatures. The inverted planar heterojunction PSCs based on SSG in our cases considerably reduce the voltage-deficit to as low as $0.41 \mathrm{~V}$. This high $V_{\text {oc }}$ rivals the regular perovskite solar cells reported by other literatures. With the exception of reference 3 , the bandgap values we quote in the table below are the Tauc bandgap s produced from Tauc plots form the absorption spectrum of the perovskite absorber layers. For reference 3 please see the note below.*

\begin{tabular}{|c|c|c|c|c|c|c|c|}
\hline $\begin{array}{l}\text { Metal-halide } \\
\text { perovskite }\end{array}$ & $\begin{array}{l}\mathrm{PCE} \\
(\%)\end{array}$ & $\begin{array}{c}J_{s c} \\
\left.(\mathrm{~mA} \mathrm{~cm})^{-2}\right)\end{array}$ & $\mathrm{FF}$ & $\begin{array}{l}V_{\mathrm{oc}} \\
(\mathrm{V})\end{array}$ & Bandgap & $\begin{array}{l}\text { Voltage } \\
\text { deficit }\end{array}$ & Literatures \\
\hline $\mathrm{MAPbI}_{3}$ & 20.10 & 23.51 & 0.830 & 1.03 & $1.59 \mathrm{eV}$ & $0.56 \mathrm{~V}$ & Ref. (47) \\
\hline $\mathrm{MAPbI}_{3}$ & 19.23 & 21.79 & 0.795 & 1.11 & $1.59 \mathrm{eV}$ & $0.48 \mathrm{~V}$ & Ref. (48) \\
\hline $\mathrm{MAPbI}_{3}$ & 20.78 & 24.00 & 0.780 & 1.11 & $1.59 \mathrm{eV}$ & $0.48 \mathrm{~V}$ & Ref. (2) \\
\hline $\mathrm{MAPbI}_{3}$ & 19.40 & 22.60 & 0.750 & 1.13 & $1.59 \mathrm{eV}$ & $0.46 \mathrm{~V}$ & Ref. (20) \\
\hline $\begin{array}{l}\left(\mathrm{FAPbI}_{3}\right)_{0.85}(\mathrm{MA} \\
\left.\mathrm{PbBr}_{3}\right)_{0.15}\end{array}$ & 20.59 & 22.99 & 0.791 & 1.13 & $1.59 \mathrm{eV}$ & $0.46 \mathrm{~V}$ & Ref. (3) \\
\hline $\begin{array}{l}\left(\mathrm{FA}_{0.95} \mathrm{PbI}_{2.95}\right)_{0.85}( \\
\left.\mathrm{MAPbBr}_{3}\right)_{0.15}\end{array}$ & 21.51 & 22.50 & 0.790 & 1.21 & $1.62 \mathrm{eV}$ & $0.41 \mathrm{~V}$ & This work \\
\hline
\end{tabular}

* Reference 3 did not show any absorption data for the perovskite absorber layers used in their study, nor any spectral response data for their highest open-circuit voltage device. The only spectral data available is the normalized EQE plot from the certified cell data presented in fig. S6 of reference 3 , and the cell gave a $V_{\text {oc }}$ of $1.13 \mathrm{~V}$. We have determined the bandgap from this EQE plot to be $1.59 \mathrm{eV}$, via the differential of the $\mathrm{EQE}$ spectrum (fig. S6), leading to a voltage-deficit of $0.46 \mathrm{~V}$. 


\section{Table S8.}

Solar cell performance parameters obtained from the devices based on various electronextraction layers, buffer layers, and metal electrodes. The devices were measured from reverse scans (RS, from $1.22 \mathrm{~V}$ to $-0.02 \mathrm{~V}$ ) under simulated AM $1.5 \mathrm{G}$ solar irradiation at $100 \mathrm{~mW} \mathrm{~cm}^{-2}$.

\begin{tabular}{|c|c|c|c|c|c|c|c|}
\hline ESL & SSG & $\begin{array}{l}\text { Buffer } \\
\text { layers }\end{array}$ & M & $\begin{array}{l}V_{\mathrm{oc}} \\
(\mathrm{V})\end{array}$ & $\begin{array}{c}J_{s c} \\
\left(\mathrm{~mA} \mathrm{~cm}{ }^{-2}\right)\end{array}$ & $\mathrm{FF}$ & $\begin{array}{l}\text { PCE } \\
(\%)\end{array}$ \\
\hline ITIC & SSG-G & $\mathrm{BCP}$ & $\mathrm{Cu}$ & $1.20 \pm 0.02$ & $21.65 \pm 0.93$ & $0.65 \pm 0.02$ & $16.89 \pm 0.65$ \\
\hline ITIC & SSG-F & BCP & $\mathrm{Cu}$ & $1.19 \pm 0.02$ & $22.34 \pm 0.96$ & $0.63 \pm 0.03$ & $16.75 \pm 0.58$ \\
\hline $\mathrm{PC}_{61} \mathrm{BM}$ & SSG-G & $\mathrm{BCP}$ & $\mathrm{Cu}$ & $1.20 \pm 0.01$ & $22.26 \pm 0.97$ & $0.78 \pm 0.02$ & $20.84 \pm 0.46$ \\
\hline $\mathrm{PC}_{61} \mathrm{BM}$ & SSG-G & $\mathrm{BCP}$ & $\mathrm{Ag}$ & $1.20 \pm 0.01$ & $22.47 \pm 0.98$ & $0.77 \pm 0.02$ & $20.76 \pm 0.47$ \\
\hline $\mathrm{PC}_{61} \mathrm{BM}$ & SSG-G & BCP & $\mathrm{Au}$ & $1.20 \pm 0.02$ & $21.96 \pm 0.87$ & $0.74 \pm 0.04$ & $19.50 \pm 0.67$ \\
\hline $\mathrm{PC}_{61} \mathrm{BM}$ & SSG-G & ITO & $\mathrm{Cu}$ & $1.20 \pm 0.01$ & $22.34 \pm 0.75$ & $0.69 \pm 0.02$ & $18.50 \pm 1.12$ \\
\hline $\mathrm{PC}_{61} \mathrm{BM}$ & SSG-G & ITO & $\mathrm{Ag}$ & $1.18 \pm 0.02$ & $22.10 \pm 1.05$ & $0.67 \pm 0.03$ & $17.47 \pm 0.98$ \\
\hline $\mathrm{PC}_{61} \mathrm{BM}$ & SSG-G & ITO & $\mathrm{Au}$ & $1.19 \pm 0.03$ & $22.51 \pm 0.94$ & $0.65 \pm 0.03$ & $17.41 \pm 1.15$ \\
\hline $\mathrm{PC}_{61} \mathrm{BM}$ & SSG-G & $\mathrm{CrO}_{\mathrm{x}}$ & $\mathrm{Cu}$ & $1.18 \pm 0.03$ & $22.31 \pm 0.99$ & $0.71 \pm 0.04$ & $18.69 \pm 0.97$ \\
\hline $\mathrm{PC}_{61} \mathrm{BM}$ & SSG-G & $\mathrm{CrO}_{\mathrm{x}}$ & $\mathrm{Ag}$ & $1.19 \pm 0.02$ & $22.23 \pm 1.02$ & $0.68 \pm 0.02$ & $17.99 \pm 0.96$ \\
\hline $\mathrm{PC}_{61} \mathrm{BM}$ & SSG-G & $\mathrm{CrO}_{\mathrm{x}}$ & $\mathrm{Au}$ & $1.19 \pm 0.01$ & $22.16 \pm 1.13$ & $0.67 \pm 0.02$ & $17.67 \pm 1.10$ \\
\hline
\end{tabular}




\section{References}

1. K. A. Bush et al., 23.6\%-efficient monolithic perovskite/silicon tandem solar cells with improved stability. Nat. Energy 2, 17009 (2017).

2. J. Zhao et al., Is $\mathrm{Cu}$ a stable electrode material in hybrid perovskite solar cells for a 30-year lifetime? Energy Environ. Sci. 9, 3650-3656 (2016).

3. X. Zheng et al., Defect passivation in hybrid perovskite solar cells using quaternary ammonium halide anions and cations. Nat. Energy 2, 17102 (2017).

4. W. Chen et al., Efficient and stable large-area perovskite solar cells with inorganic charge extraction layers. Science 350, 944-948 (2015).

5. M. Saliba et al., Incorporation of rubidium cations into perovskite solar cells improves photovoltaic performance. Science 354, 206-209 (2016).

6. L. Ling et al., Precisely controlled hydration water for performance improvement of organicinorganic perovskite solar cells. Adv. Funct. Mater. 26, 5028-5034 (2016).

7. J. Huang, Y. Yuan, Y. Shao, Y. Yan, Understanding the physical properties of hybrid perovskites for photovoltaic applications. Nat. Rev. Mater. 2, 17042 (2017).

8. S. Chen et al., Exploring the limiting open-circuit voltage and the voltage loss mechanism in planar $\mathrm{CH}_{3} \mathrm{NH}_{3} \mathrm{PbBr}_{3}$ perovskite solar cells. Adv. Energy Mater. 6, 1600132 (2016).

9. W. Zhang, G. E. Eperon, H. J. Snaith, Metal halide perovskites for energy applications. Nat. Energy 1, 16048 (2016).

10. D. W. deQuilettes et al., Impact of microstructure on local carrier lifetime in perovskite solar cells. Science 348, 683-686 (2015).

11. G. Han et al., Facile method to reduce surface defects and trap densities in perovskite photovoltaics. ACS Appl. Mater. Interfaces 9, 21292-21297 (2017).

12. K. T. Cho et al., Highly efficient perovskite solar cells with a compositionally engineered perovskite/hole transporting material interface. Energy Environ. Sci. 10, 621-627 (2017).

13. N. De Marco et al., Guanidinium: a route to enhanced carrier lifetime and open-circuit voltage in hybrid perovskite solar cells. Nano Lett. 16, 1009-1016 (2016).

14. D. Bi et al., Efficient luminescent solar cells based on tailored mixed-cation perovskites. Sci. Adv. 2, e1501170 (2016).

30 15. D. Shi et al., Low trap-state density and long carrier diffusion in organolead trihalide perovskite single crystals. Science 347, 519-522 (2015).

16. F. Deschler et al., High photoluminescence efficiency and optically pumped lasing in solution-processed mixed halide perovskite semiconductors. J. Phys. Chem. Lett. 5, 1421-1426 (2014).

35 17. G. E. Eperon, D. Moerman, D. S. Ginger, Anticorrelation between local photoluminescence and photocurrent suggests variability in contact to active layer in perovskite solar cells. ACS Nano 10, 10258-10266 (2016).

18. M. Yang et al., Facile fabrication of large-grain $\mathrm{CH}_{3} \mathrm{NH}_{3} \mathrm{PbI}_{3-\mathrm{x}} \mathrm{Br}_{\mathrm{x}}$ films for high-efficiency solar cells via $\mathrm{CH}_{3} \mathrm{NH}_{3} \mathrm{Br}-$ selective Ostwald ripening. Nat. Commun. 7, 12305 (2016).

40 19. W. Nie, et al., High-efficiency solution-processed perovskite solar cells with millimeterscale grains. Science 347, 522-525 (2015).

20. Y. Shao, Y. Yuan, J. Huang, Correlation of energy disorder and open-circuit voltage in hybrid perovskite solar cells. Nat. Energy 1, 15001 (2016).

21. Y. Wu et al., Perovskite solar cells with $18.21 \%$ efficiency and area over $1 \mathrm{~cm}^{2}$ fabricated by heterojunction engineering. Nat. Energy 1, 16148 (2016). 
22. M. Liu, M. B. Johnston, H. J. Snaith, Efficient planar heterojunction perovskite solar cells by vapour deposition. Nature 501, 395-398 (2013).

23. T. Leijtens et al., Electronic properties of meso-superstructured and planar organometal halide perovskite films: charge trapping, photodoping, and carrier mobility. ACS Nano $\mathbf{8}$, 7147-7155 (2014).

24. X. Zhang et al., Bright perovskite nanocrystal films for efficient light-emitting devices. $J$. Phys. Chem. Lett. 7, 4602-4610 (2016).

25. P. Schulz et al., Electronic level alignment in inverted organometal perovskite solar cells. Adv. Mater. Interfaces 2, 1400532 (2015).

26. Y. C. Kim et al., Beneficial effects of $\mathrm{PbI}_{2}$ incorporated in organo-lead halide perovskite solar cells. Adv. Energy Mater. 6, 1502104 (2016).

27. Q. Chen et al., Controllable self-induced passivation of hybrid lead iodide perovskites toward high performance solar cells. Nano Lett. 14, 4158-4163 (2014).

28. K. Tvingstedt et al., Radiative efficiency of lead iodide based perovskite solar cells. Sci. Rep. 4, 6071 (2014).

29. C. Quarti, F. D. Angelis, D. Beljonne, Influence of surface termination on the energy level alignment at the $\mathrm{CH}_{3} \mathrm{NH}_{3} \mathrm{PbI}_{3}$ Perovskite/ $\mathrm{C}_{60}$ interface. Chem. Mater. 29, 958-968 (2017).

30. J. J. Li et al., Microscopic investigation of grain boundaries in organolead halide perovskite solar cells. ACS Appl. Mater. Interfaces 7, 28518-28523 (2015).

31. C. Bi et al., Non-wetting surface-driven high-aspect-ratio crystalline grain growth for efficient hybrid perovskite solar cells. Nat. Commun., 6, 7747 (2015).

32. M. B. Price et al., Hot-carrier cooling and photoinduced refractive index changes in organicinorganic lead halide perovskites. Nat. Commun. 6, 8420 (2015).

33. X. Hou et al., Effect of guanidinium on mesoscopic perovskite solar cells. J. Mater. Chem. A 5, 73-78 (2017).

34. S. D. Stranks et al., Recombination kinetics in organic-inorganic perovskites: excitons, free charge, and subgap states. Phys. Rev. Applied 2, 034007 (2014).

35. J. Burschka et al., Sequential deposition as a route to high-performance perovskite-sensitized solar cells. Nature 499, 316-319 (2013).

30 36. A. D. Jodlowski et al., Large guanidinium cation mixed with methylammonium in lead iodide perovskites for 19\% efficient solar cells. Nat. Energy 2, 972-979 (2017).

37. N. J. Jeon et al., Compositional engineering of perovskite materials for high-performance solar cells. Nature 517, 476-480 (2015).

38. J. C. de Mello, H. F. Wittmann, R. H. Friend, An improved experimental determination of external photoluminescence quantum efficiency. Adv. Mater. 9, 230-232 (1997).

39. A. Sadhanala et al., Preparation of single-phase films of $\mathrm{CH}_{3} \mathrm{NH}_{3} \mathrm{~Pb}\left(\mathrm{I}_{1-\mathrm{x}} \mathrm{Br}_{\mathrm{x}}\right)_{3}$ with sharp optical band edges. J. Phys. Chem. Lett. 5, 2501-2505 (2014).

40. S. Pathak et al., Atmospheric influence upon crystallization and electronic disorder and its impact on the photophysical properties of organic-inorganic perovskite solar cells. ACS Nano 9, 2311-2320 (2015).

41. I. Robinson, R. Harder, Coherent X-ray diffraction imaging of strain at the nanoscale. Nat. Mater. 8, 291-298 (2009).

42. A. Pramanick et al., Microdomain dynamics in single-crystal $\mathrm{BaTiO}_{3}$ during paraelectricferroelectric phase transition measured with time-of-flight neutron scattering. Phys. Rev. B 92, 174103 (2015). 
43. G. K. Williamson, W. H. Hall, X-ray line broadening from filed aluminium and wolfram. Acta Metall. 1, 22-31 (1953).

44. Y. Zhao, J. Zhang, Microstrain and grain-size analysis from diffraction peak width and graphical derivation of high-pressure thermomechanics. J. Appl. Cryst. 41, 1095-1108 (2008).

45. U. Rau, Reciprocity relation between photovoltaic quantum efficiency and electroluminescent emission of solar cells. Phys. Rev. B 76, 085303 (2007).

46. J. P. Correa-Baena et al., Promises and challenges of perovskite solar cells. Science 358, 739-744 (2017).

47. C. H. Chiang, M. K. Nazeeruddin, M. Grätzel, C. G. Wu, The synergistic effect of $\mathrm{H}_{2} \mathrm{O}$ and DMF towards stable and 20\% efficiency inverted perovskite solar cells. Energy Environ. Sci. 10, 808-817 (2017).

48. W. Chen et al., Cesium doped $\mathrm{NiO}_{\mathrm{x}}$ as an efficient hole extraction layer for inverted planar perovskite solar cells. Adv. Energy Mater. 7, 1700722 (2017). 